SINGLE AND DOUBLE LAYER POTENTIALS ON DOMAINS WITH CONICAL POINTS I: STRAIGHT CONES

By

Victor Nistor

and

Yu Qiao

IMA Preprint Series \# 2358

( January 2011)

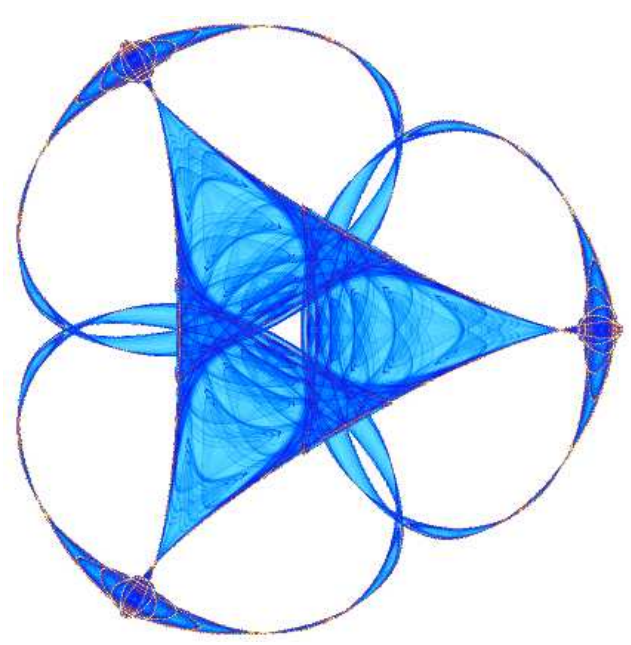

INSTITUTE FOR MATHEMATICS AND ITS APPLICATIONS

UNIVERSITY OF MINNESOTA 400 Lind Hall

207 Church Street S.E.

Minneapolis, Minnesota 55455-0436

Phone: 612-624-6066 Fax: 612-626-7370

URL: http://www.ima.umn.edu 


\title{
SINGLE AND DOUBLE LAYER POTENTIALS ON DOMAINS WITH CONICAL POINTS I: STRAIGHT CONES
}

\author{
VICTOR NISTOR AND YU QIAO
}

\begin{abstract}
Let $\Omega=\mathbb{R}^{+} \omega$ be an open cone in $\mathbb{R}^{n}, n \geqslant 3$, where $\omega \subset S^{n-1}$ is a smooth subdomain of the unit sphere. Denote by $K$ and $S$ the double and single layer potential operators associated to $\Omega$ and the Laplace operator $\Delta$. Let $r$ be the distance to the origin. We consider a natural class of operators on $\partial \Omega$, called Mellin convolution operators with good mapping properties between weighted Sobolev spaces and show that $r^{a} K r^{-a}$ and $r^{b-\frac{1}{2}} \mathrm{Sr}^{-b-\frac{1}{2}}$ are Mellin convolution operators for $a \in(-1, n-1)$ and $b \in\left(\frac{1}{2}, n-\frac{3}{2}\right)$. This result allows us to prove that $\frac{1}{2} \pm K$ and $r^{-\frac{1}{2}} S r^{-\frac{1}{2}}$ are invertible between suitable weighted Sobolev spaces. Classically, it is known that the invertibility of these operators gives solvability results in weighted Sobolev spaces for the Dirichlet problem on $\Omega$.
\end{abstract}

\section{INTRODUCTION}

The Dirichlet problem for Laplace's equation in a sufficiently regular bounded domain $\Omega \subset \mathbb{R}^{n}$ can be solved for continuous boundary values using the double layer potential operator

$$
u(x)=K \varphi(x)=-\frac{1}{\omega_{n}} \int_{\partial \Omega} \frac{(x-y) \cdot \nu(y)}{|x-y|^{n}} \varphi(y) d S_{n-1}(y),
$$

or the single layer potential operator

$$
v(x)=S \varphi(x)=\frac{1}{(n-2) \omega_{n}} \int_{\partial \Omega} \frac{1}{|x-y|^{n-2}} \varphi(y) d S_{n-1}(y) .
$$

Potential theory can be traced back to the works of Lagrange, Laplace, and Poisson. One of the main motivations to study the layer potential operators in the setting of potential theory is that the single and double layer potentials represent a harmonic function in terms of its boundary data. Moreover, the layer potentials play a fundamental role in many real-world systems, especially in physics. For instance, Gauss used single layer potential to find, for arbitrary conductor $\Omega$, the equilibrium charge distribution with the total charge $M$. (See [26] for a more detailed historical account.) Many other mathematicians made important contributions to potential theory, such as Liouville, Neumann, Poincaré, Fredholm, Hilbert, and many others.

Recently, the method of potential theory has attracted some attention both in the theoretical and the applied mathematics. For instance, it plays an important role in solving boundary value problems of elliptic equations, the Helmholtz equation, the equation of linear elasticity. It has applications to electromagnetic scatering [29]. In applied mathematics, the so-called boundary element methods are often used

Date: June 27, 2011.

Nistor was partially supported by the NSF Grants DMS-0713743, OCI-0749202, and DMS1016556. Manuscripts available from http://www.math.psu.edu/nistor/. 
for domains with singularities. The advantage of this method is that it decreases the dimension of the discretization space. For instance, Bremer and Rokhin [2] develop a numerical procedure for the construction of quadrature formulae suitable for the efficient discretization of boundary integral equations on two-dimensional polygonal domains.

A great many number of papers and books have been written on the method of layer potentials. It is not possible to mention all of them here, but we want to mention a few, beginning with the books by Courant and Hilber [4], Foland [10], Kress [20], McLean [26], and Taylor [35], which give a rather complete account of the theory on smooth domains, which is by now fairly well understood. We are more interested in non-smooth domains. There are also many papers devoted to the method of layer potentials on non-smooth domains. These works can be roughly divided into two categories: works devoted to Lipschitz domains and works devoted to polyhedral domains (mostly polygonal). The case of Lipschitz domains is by far the most studied among non-smooth domains, and is also fairly well understood. We would like to mention here the papers of Jerrison and Kenig [12, 13, 14], Kenig [17], Kenig and Pipher [18], and Verchota [37] that give rather complete resuls for domains in the euclidean space. The works of D. Mitrea and I. Mitrea [28], I. Mitrea and M. Mitrea [31], M. Mitrea and Taylor [33, 34], and Taylor [36], in which the method of layer potentials is applied to Lipschitz domains on manifolds. See also Costabel's paper [3] for a nice introduction to the method of layer potentials using more elementary methods. See also [9] for earlier results on $\mathcal{C}^{1}$-domains. In this paper, we are more interested in polyhedral domains. By comparison, much fewer works were devoted to this case. We want to mention however the papers of I. Mitrea [30], Lewis [22], Lewis and Parenti [23] for work on polygonal domains. The papers of Elschner [6], Fabes, Jodeit, Lewis [8], Verchota and Vogel [38] deal with the case of polyhedral domains in three and four dimensions. See also [16] for the related case of interface problems. See also the references contained in those papers.

In our paper, we are interested in higher dimensional domains, but with the simplest type of singularities, namely conical singularities. We found few related paper using layer potentials $[24,32]$. We want to mention, however, also the work of Kondratiev on boundary value problems on domains with conical points as well as the books of Egorov and Schulze [5], Kapanadze and Schulze [15], Mazya and Rossmann [25], and Melrose [27].

The virtue of using layer potential operators is that they reduce the existence problem for the Laplace equation to the invertibility of either of the operators $\frac{1}{2}+K$ or $S$. For instance, if the boundary is $\mathcal{C}^{2}$, then we can apply the classical Fredholm theory to the operators $\frac{1}{2}+K$ and $S$ to solve the Laplace's equation when $\varphi \in L^{2}(\partial \Omega)$ or $\varphi \in \mathcal{C}(\partial \Omega)[10,20]$. If the boundary is only $\mathcal{C}^{1}$, the operator $K$ is still compact [9] on $L^{2}(\partial \Omega)$. However, if the boundary of our domain $\Omega$ is not $\mathcal{C}^{1}$, which is the case, for instance, if $\Omega$ is a polygon or, more generally, a domain with conical points, the double layer potential operator is no longer compact $[6,8,19,20,22,23,28,30,32,35]$. A similar approach applies also to the Neumann problem for the Laplace equation.

These results justify studying in detail domains with conical points. For obvious technical reasons, we found it necessary to first take a close look at the case of 
straight cones, which is the main topic of this paper. We need a thorough understanding of the case of straight cones to be able to handle successfully the case of domains with conical points. In addition to using the theory of compact and Fredholm operators, to study straight cones, we found it necessary to use techniques of harmonic analysis such as the Mellin transform. Our main results are as follows. First, we provide a reduction, via Mellin transform, of the study of layer potentials on cones to the study of a family layer potential operators on the basis of the cone. This allows us to establish the invertibility of the relevant layer potential operators on cones by reducing to the pointwise invertibility of a family of operators on the boundary of the basis of the cone. (It is known that for the type of operators that we consider, the pointwise invertibility is enough for global invertibility $[5,27]$, but since this may be a more subtle point, we have included a proof using Banach Algebras.) This invertibility turns out to hold for a range of weights of our Sobolev spaces. In the process, we also determine in detail the structure of the layer potentials in terms of operator valued Mellin convolution operators.

This paper is organized as follows. In Section 1, we introduce the single and double layer potentials for sufficiently regular domains. In Section 2 , we define Mellin convolution operators and the filtered algebra $\mathcal{R}:=\cup \mathcal{R}_{-k}$ of Mellin convolution pseudodifferential operators on $\partial \Omega$, with $\mathcal{R}_{k}$ denoting the operators of order $k$. We then prove mapping properties for operators in $\mathcal{R}_{k}$. In Section 3, we introduce the Mellin transform for operators in $\mathcal{R}$ and study its properties. In Section 4 and Section 5, we define the double and single layer potential operators $K$ and $S$ associated to the Laplace operator on a straight cone, and related operator $S_{0}=M_{r^{-\frac{1}{2}}} S_{0} M_{r^{-\frac{1}{2}}}$, which is unlike $S$, turns out to be a Mellin convolution operator. For the purpose of working with a dilation invariant measure on the cone, we introduce the modified operators $\widetilde{K}:=M_{r^{\frac{n-1}{2}}} K M_{r^{-\frac{n-1}{2}}}$ and $\widetilde{S}_{0}:=M_{r^{\frac{n-2}{2}}} S_{0} M_{r^{-\frac{n-2}{2}}}$. In Section 6 , we define weighted Sobolev spaces on $\Omega$ or on $\partial \Omega$. Then we prove that $\widetilde{K}$ and $\widetilde{S}_{0}$ belong to $\mathcal{R}_{-1}$. Using general results in Section 2, we establish some mapping properties of $K$ and $S$ between some weighted Sobolev spaces. Lastly Section 7 contains the proofs of our main results: that the operators $\frac{1}{2}+K$ and $S_{0}$ are isomorphisms between weighted Sobolev spaces for suitable weights.

We thank Irina Mitrea for some useful comments.

\section{LAYER POTENTIALS}

Let us fix a Riemannian manifold $M$ without boundary. The single and double layer potentials are defined for any domain $\Omega \subset M$ whose boundary is sufficiently regular, but not necessarily smooth. These layer potentials are associated to a positive, strongly elliptic operator $P=\sum a_{i j} \partial_{i} \partial_{j}+\sum b_{j} \partial_{j}+c$ and a fundamental solution $E(x, y)$ of $P$. In this paper, we just focus on the case where $P$ is the positive Laplacian: $P=-\Delta$ and $E$ is its usual fundamental solution.

We shall denote by $\Psi^{k}(M)$ the space of pseudodifferential operators of order $k$ on $M[10,11,36]$. Suppose we are given a domain $\Omega \subset M$ on which there is a given fundamental solution $E(x, y)$ of the Laplacian. Let $\omega_{n}$ denote the area of $S^{n-1}$ and let $c_{n}=-\left[(n-2) \omega_{n}\right]^{-1}$ for $n \geqslant 3$, and $c_{2}=1 / 2 \pi$. More precisely, we assume that we are given a smooth function $E: M \times M \backslash \operatorname{diag}_{M} \rightarrow \mathbb{R}$, where $\operatorname{diag}_{M}$ is the diagonal of $M$, such that for any fixed $y, E(x, y)$ defines a distribution in $x$ 
satisfying

$$
-\Delta_{x} E(x, y)=\delta_{y}(x)
$$

where $E(x, y)$ is the Schwartz kernel of an operator $E(x, D) \in \Psi^{-2}(M)$ and $\delta_{y}$ is the Dirac distribution at $y$. We then have

$$
E(x, y) \sim c_{n} \operatorname{dist}(x, y)^{2-n}+\cdots
$$

as $x \rightarrow y$, if $n \geqslant 3$, while

$$
E(x, y) \sim c_{2} \log \operatorname{dist}(x, y)+\cdots
$$

if $n=2$. See $[7,36]$.

Assume $\Omega$ is a polyhedral domain, so that the surface measure $d S_{n-1}$ on $\Omega$ is defined and that the unit normal vector $\nu(y)$ to $\partial \Omega$ is defined almost everywhere with respect to $d S_{n-1}$. We introduce the operators $\mathcal{S}$ and $\mathcal{D}$, mapping functions on the boundary $\partial \Omega$ to functions on the whole space $\mathbb{R}^{n}$. Let $\nu$ denote the unit outer normal vector field on $\partial \Omega$. For any integrable function $f: \partial \Omega \rightarrow \mathbb{C}$ and for any $x \in M \backslash \partial \Omega$, we define

$$
\mathcal{S} f(x)=\int_{\partial \Omega} E(x, y) f(y) d S_{n-1}(y)
$$

and

$$
\mathcal{D} f(x)=\int_{\partial \Omega} \frac{\partial E}{\partial \nu_{y}}(x, y) f(y) d S_{n-1}(y) .
$$

Similarly, for $x \in \partial \Omega$, we define

$$
S f(x)=S(\Delta, E ; \Omega) f(x)=\int_{\partial \Omega} E(x, y) f(y) d S_{n-1}(y)
$$

and

$$
K f(x)=K(\Delta, E ; \Omega)=\int_{\partial \Omega} \frac{\partial E}{\partial \nu_{y}}(x, y) f(y) d S_{n-1}(y) .
$$

In the cases of interest, we will check that these integrals converge.

The operators $K=K(\Delta, E ; \Omega)$ and $S=S(\Delta, E ; \Omega)$ define the double and single layer potential operators for $\Delta, E$, and $\Omega$. They map functions on $\partial \Omega$ to functions on $\partial \Omega$. As indicated in the notation, the operators $S$ and $K$ depend on the choice of $E$. In this paper we shall study the case when $\Omega$ is an open cone (5). In this case, we will show that $K$ and $S$ are related to some specific Mellin convolution operators, which will be used to study the properties of these operators.

\section{Mellin CONVOlution operators}

Let $\omega \subset S^{n-1}(n \geqslant 3)$ be an open subset with smooth boundary. We allow $\omega$ to be disconnected. Denote by

$$
\Omega:=\mathbb{R}^{+} \omega:=\left\{t y^{\prime}: y^{\prime} \in \omega, t \in(0, \infty)\right\}
$$

the open cone with base $\omega$. Since any point $x \in \Omega$ can uniquely be written as $r x^{\prime}$, where $r \in \mathbb{R}^{+}$and $x^{\prime} \in \omega$, we shall sometimes identify a point $x=r x^{\prime} \in \Omega$ with 
the pair $\left(r, x^{\prime}\right) \in \mathbb{R}^{+} \times \omega$. Denote the surface measure on $\omega$ by $d S_{n-1}\left(x^{\prime}\right)$ and the measure on $\partial \omega$ by $d S_{n-2}\left(x^{\prime}\right)$, and set

$$
d \mu_{n}\left(r, x^{\prime}\right)=r^{-1} d r d S_{n-1}\left(x^{\prime}\right), \text { and } d \mu_{n-1}\left(r, x^{\prime}\right)=r^{-1} d r d S_{n-2}\left(x^{\prime}\right),
$$

which give rise to Hilbert spaces $L^{2}\left(\Omega, d \mu_{n}\right)$ and $L^{2}\left(\partial \Omega, d \mu_{n-1}\right)$.

Definition 2.1. Let $p=p\left(r, x^{\prime}, y^{\prime}\right) \in \mathcal{C}^{\infty}\left(\mathbb{R}^{+} \times \omega \times \omega\right)$ and $u \in \mathcal{C}_{c}(\Omega)$. Define the function $P u$ on $\Omega$ by

$$
P u\left(r, x^{\prime}\right)=p * u\left(r, x^{\prime}\right)=\int_{\omega} \int_{0}^{\infty} p\left(r / s, x^{\prime}, y^{\prime}\right) u\left(s, y^{\prime}\right) \frac{d s}{s} d S_{n-1}\left(y^{\prime}\right) .
$$

The operator $C_{p}:=P$ will be called the smoothing Mellin convolution operator on $\Omega$ with (operator valued) convolution kernel $p$. If $p$ is only continuous, the operator $C_{p}:=P$ will be called the Mellin convolution operator on $\Omega$ with continuous (operator valued) convolution kernel $p$.

If $p=p\left(r, x^{\prime}, y^{\prime}\right) \in \mathcal{C}^{\infty}\left(\mathbb{R}^{+} \times \partial \omega \times \partial \omega\right)$, we define smoothing Mellin convolution operators on $\partial \Omega$ in the same way. Any extension of $P$ contained in the closure of $P$ will also be called a Mellin convolution operator.

The operator valued convolution kernel of a Mellin convolution operator $P$ will be denoted by $k_{P}$. Thus $k_{P}=p$ in the above definition.

Definition 2.2. For any $t \in(0, \infty)$, we define the dilation $\alpha_{t}$ (by $t$ ) by the formula

$$
\alpha_{t} f(x)=f(t x) \text {. }
$$

It is clear that for each $t \in \mathbb{R}^{+}, \alpha_{t}$ is unitary on $L^{2}\left(\Omega, d \mu_{n}\right)$ or on $L^{2}\left(\partial \Omega, d \mu_{n-1}\right)$.

Lemma 2.3. Let $P$ be a bounded integral operator on $L^{2}\left(\Omega, d \mu_{n}\right)$ with continuous integral kernel $p_{1}\left(r, s, x^{\prime}, y^{\prime}\right)$. Then $P$ is a Mellin convolution operator (with continuous kernel) if, and only if, it satisfies

$$
P \alpha_{t}=\alpha_{t} P
$$

for any $t \in \mathbb{R}^{+}$. The same is true if $P$ is a (possibly unbounded) operator on $L^{2}\left(\partial \Omega, d \mu_{n-1}\right)$.

Proof. For any $u \in \mathcal{C}_{c}(\Omega)$, we have

$$
\begin{aligned}
\left(P \alpha_{t} u\right)\left(r, x^{\prime}\right) & =\int_{\omega} \int_{0}^{\infty} p_{1}\left(r, s, x^{\prime}, y^{\prime}\right) u\left(t s, y^{\prime}\right) \frac{d s}{s} d S_{n-1}\left(y^{\prime}\right) \\
& =\int_{\omega} \int_{0}^{\infty} p_{1}\left(r, s / t, x^{\prime}, y^{\prime}\right) u\left(s, y^{\prime}\right) \frac{d s}{s} d S_{n-1}\left(y^{\prime}\right),
\end{aligned}
$$

and

$$
\left(\alpha_{t} P u\right)\left(r, x^{\prime}\right)=\int_{\omega} \int_{0}^{\infty} p_{1}\left(t r, s, x^{\prime}, y^{\prime}\right) u\left(s, y^{\prime}\right) \frac{d s}{s} d S_{n-1}\left(y^{\prime}\right) .
$$

Since $p_{1}$ is continuous, we obtain $p_{1}\left(r, s / t, x^{\prime}, y^{\prime}\right)=p_{1}\left(t r, s, x^{\prime}, y^{\prime}\right)$ for any $t \in$ $\mathbb{R}^{+}$. This show that $p_{1}\left(r, s, x^{\prime}, y^{\prime}\right)=p_{1}\left(1, r / s, x^{\prime}, y,\right)$, and hence that $p_{1}\left(r, s, x^{\prime}, y^{\prime}\right)$ depends only on $r / s$. Therefore, $P$ is a Mellin convolution operator with kernel $p\left(t, x^{\prime}, y^{\prime}\right)=p_{1}\left(1, r / s, x^{\prime}, y^{\prime}\right)$. The converse is proved in exactly the same way.

For a Mellin convolution operator $P$ on $\Omega=\mathbb{R}^{+} \omega$ with operator valued kernel $p\left(r, x^{\prime}, y^{\prime}\right)$, we shall denote by $P(r)$ the integral operator on $\omega$ with kernel $p\left(r, x^{\prime}, y^{\prime}\right)$. By differentiating with respect to $t$ in Lemma 2.3 and taking into account that $r \partial_{r}=\left.\left(\alpha_{t}\right)^{\prime}\right|_{t=0}$, we obtain the following corollary. 
Corollary 2.4. Let $P$ be a Mellin convolution operator on $\Omega$ with operator-valued convolution kernel $k_{P}\left(r, x^{\prime}, y^{\prime}\right)=p\left(r, x^{\prime}, y^{\prime}\right)$. Then

$$
\left(r \partial_{r}\right) P=P\left(r \partial_{r}\right) .
$$

Moreover, the operator $\left(r \partial_{r}\right) P$ is the Mellin convolution operator with operatorvalued convolution kernel $\left(r \partial_{r}\right) p$. The same is true if $P$ is a Mellin convolution operator on $\partial \Omega$.

We now introduce suitable algebras of Mellin convolution operators. Let $\Delta_{\partial \Omega}$ be the Laplace operator on $\mathbb{R}^{+} \times \partial \omega$ associated to the metric $\left(r^{-1} d r\right)^{2}+\left(d x^{\prime}\right)^{2}$, where $\left(d x^{\prime}\right)^{2}$ is the metric on $\partial \omega$ induced from $S^{n-1}$. Then

$$
\Delta_{\partial \Omega}=\left(r \partial_{r}\right)^{2}+\Delta_{\partial \omega}
$$

Proposition 2.5. Let $\varphi \in \mathcal{C}^{\infty}\left(\mathbb{R}^{+} \times \partial \omega \times \partial \omega\right)$ and $C_{\varphi}$ denote the Mellin convolution operator on $\partial \Omega$ with kernel $\varphi$. Then we have, for $j, k \in \mathbb{N} \cup\{0\}$,

$$
\Delta_{\partial \omega}^{j} C_{\varphi} \Delta_{\partial \omega}^{k}=C_{\psi},
$$

where $\psi\left(r, x^{\prime}, y^{\prime}\right)=\Delta_{\partial \omega, x^{\prime}}^{j} \Delta_{\partial \omega, y^{\prime}}^{k} \varphi\left(r, x^{\prime}, y^{\prime}\right)$. Also

$$
\left(r \partial_{r}\right)^{j} C_{\varphi}=C_{\varphi}\left(r \partial_{r}\right)^{j}=C_{\left(r \partial_{r}\right)^{j} \varphi} .
$$

Proof. Integration by parts implies the first statement. To prove the second statement, it is enough to show the case $j=1$. The result follows from Corollary 2.4, using also a short calculation.

Definition 2.6. Define $\mathcal{R}_{-\infty}$ to be the space of Mellin convolution operators $P$ on $\partial \Omega$ with kernel $p\left(r, x^{\prime}, y^{\prime}\right) \in \mathcal{C}^{\infty}\left(\mathbb{R}^{+} \times \partial \omega \times \partial \omega\right)$ for which there exists $\epsilon>0$ such that, for all $\left(r, x^{\prime}, y^{\prime}\right) \in \mathbb{R}^{+} \times \partial \omega \times \partial \omega$,

$$
\left(r+\frac{1}{r}\right)^{\epsilon}\left|\left(r \partial_{r}\right)^{i} \Delta_{\partial \omega, x^{\prime}}^{j} \Delta_{\partial \omega, y^{\prime}}^{k} p\left(r, x^{\prime}, y^{\prime}\right)\right| \leqslant C(i, j, k),
$$

where $C(i, j, k)$ is a constant depending on $i, j$, and $k$.

Remark 2.7. By Proposition 2.5, the above definition implies that $p$ and its all partial derivatives have rapid decay as $r \rightarrow 0$ and $r \rightarrow \infty$.

We endow $\mathcal{R}_{-\infty}$ with the convolution product:

$$
p_{1} * p_{2}\left(r, x^{\prime}, y^{\prime}\right)=\int_{\partial \omega} \int_{0}^{\infty} p_{1}\left(r / s, x^{\prime}, z^{\prime}\right) p_{2}\left(s, z^{\prime}, y^{\prime}\right) \frac{d s}{s} d S_{n-1}\left(z^{\prime}\right) .
$$

We define an involution on $\mathcal{R}_{-\infty}$ by

$$
p^{*}\left(r, x^{\prime}, y^{\prime}\right)=p\left(r^{-1}, y^{\prime}, x^{\prime}\right) .
$$

Lemma 2.8. The space $\mathcal{R}_{-\infty}$ is a *-algebra.

Proof. If $p \in \mathcal{R}_{-\infty}$, it is easy to see that $p^{*} \in \mathcal{R}_{-\infty}$. We need to show that if $p_{1}, p_{2} \in \mathcal{R}_{-\infty}$, then $p_{1} * p_{2} \in \mathcal{R}_{-\infty}$. Let $\epsilon>0$ be the constant defining $p_{1}$ and $p_{2}$. It is enough to check that

$$
\left(r+\frac{1}{r}\right)^{\epsilon^{\prime}}\left|\left(r \partial_{r}\right)^{i} \Delta_{\partial \omega, x^{\prime}}^{j} \Delta_{\partial \omega, y^{\prime}}^{k} p_{1} * p_{2}\left(r, x^{\prime}, y^{\prime}\right)\right| \leqslant C\left(\epsilon^{\prime}, i, j, k\right),
$$


for any $0<\epsilon^{\prime}<\epsilon$. Indeed, we have

$$
\begin{aligned}
& \left(r+\frac{1}{r}\right)^{\epsilon^{\prime}}\left|\left(r \partial_{r}\right)^{i} \Delta_{\partial \omega, x^{\prime}}^{j} \Delta_{\partial \omega, y^{\prime}}^{k} p_{1} * p_{2}\left(r, x^{\prime}, y^{\prime}\right)\right| \leqslant \\
& \left(r+\frac{1}{r}\right)^{\epsilon^{\prime}} \int_{\partial \omega} \int_{0}^{\infty}\left|\left(r \partial_{r}\right)^{i} \Delta_{\partial \omega, x^{\prime}}^{j} p_{1}\left(r / s, x^{\prime}, z^{\prime}\right) \Delta_{\partial \omega, y^{\prime}}^{k} p_{2}\left(s, z^{\prime}, y^{\prime}\right)\right| \frac{d s}{s} d S_{n-1}\left(z^{\prime}\right) \\
& \leqslant C\left(r+\frac{1}{r}\right)^{\epsilon^{\prime}} \int_{\partial \omega} \int_{0}^{\infty}\left(\frac{r}{s}+\frac{s}{r}\right)^{-\epsilon}\left(s+\frac{1}{s}\right)^{-\epsilon} \frac{d s}{s} d S_{n-1}\left(z^{\prime}\right) \\
& \leqslant C \int_{0}^{\infty}\left(s+\frac{1}{s}\right)^{\epsilon^{\prime}-\epsilon} \frac{d s}{s} \leqslant C\left(\epsilon^{\prime}, i, j, k\right),
\end{aligned}
$$

The proof is now complete.

Let us define now $\Psi_{\text {prop }}^{m}(M)$ to be the set of properly supported pseudodifferential operators on a manifold $M$. Then we let

$$
\mathcal{R}_{m}:=\mathcal{R}_{-\infty}+\Psi_{\text {prop }}^{m}\left(\mathbb{R}^{+} \times \partial \omega\right)^{\mathbb{R}^{+}},
$$

where $\Psi_{\text {prop }}^{m}\left(\mathbb{R}^{+} \times \partial \omega\right)^{\mathbb{R}^{+}}$denotes dilation invariant pseudodifferential operators in $\Psi_{\text {prop }}^{m}\left(\mathbb{R}^{+} \times \partial \omega\right)$. That is, we augment the spaces of properly supported, dilation invariant pseudodifferential operators on $\mathbb{R}^{+} \times \partial \omega$ with the subspace $\mathcal{R}_{-\infty}$ of regularizing Mellin operators. Then $\mathcal{R}_{j} \mathcal{R}_{i} \subset \mathcal{R}_{i+j}$ and each $\mathcal{R}_{k}$ consists of dilation invariant operators with suitable mapping properties between weighted Sobolev spaces.

Recall that $\Omega \subset \mathbb{R}^{n}$ is an open cone, so that $\partial \Omega$ has dimension $n-1$.

Lemma 2.9. If $P \in \mathcal{R}_{-n}$ and $f \in L^{2}\left(\mathbb{R}^{+} \times \partial \omega\right)$, then

$$
\|P f\|_{L^{2}\left(\mathbb{R}^{+} \times \partial \omega\right)} \leqslant C\|f\|_{L^{2}\left(\mathbb{R}^{+} \times \partial \omega\right)}
$$

where $C$ is a constant depending on $P$ but not on $f$.

Proof. Let $p \in \mathcal{C}(\partial \Omega \times \partial \Omega)$ be the distribution kernel of $P$. Since $P$ is dilation invariant and has a continuous kernel, we can use Lemma 2.3 to conclude that it is a Mellin convolution operator.

Let us split $P=Q_{0}+Q_{1}$, where $Q_{0} \in \Psi_{\text {prop }}^{-n}(\partial \Omega)^{\mathbb{R}^{+}}$and $Q_{1} \in \mathcal{R}_{-\infty}$. Since $Q_{0}$ is properly supported, the decay properties of $p$ will depend only on the decay properties of the kernel of $Q_{1}$, and hence there exists $\epsilon>0$ such that $(r+1 / r){ }^{\epsilon} p\left(r, x^{\prime}, y^{\prime}\right)$ is bounded. Let us consider the functions

$$
H\left(x^{\prime}\right)=\int_{\partial \omega} \int_{0}^{\infty}\left|p\left(r, x^{\prime}, y^{\prime}\right)\right| \frac{d r}{r} d S_{n-2}\left(y^{\prime}\right)
$$

and

$$
J\left(y^{\prime}\right)=\int_{\partial \omega} \int_{0}^{\infty}\left|p\left(r, x^{\prime}, y^{\prime}\right)\right| \frac{d r}{r} d S_{n-2}\left(x^{\prime}\right),
$$

which will be bounded on $\partial \omega$. We have

$$
\begin{aligned}
\sup _{\left(t, x^{\prime}\right) \in \mathbb{R}^{+} \times \partial \omega} \int_{\mathbb{R}^{+} \times \partial \omega} & \left|p\left(t / s, x^{\prime}, y^{\prime}\right)\right| \frac{d s}{s} d S_{n-2}\left(y^{\prime}\right) \\
= & \sup _{x^{\prime} \in \partial \omega} \int_{\mathbb{R}^{+} \times \partial \omega}\left|p\left(s, x^{\prime}, y^{\prime}\right)\right| \frac{d s}{s} d S_{n-2}\left(y^{\prime}\right)=\sup _{x^{\prime} \in \partial \omega} H\left(x^{\prime}\right)<C .
\end{aligned}
$$


Similarly,

$$
\sup _{\left(s, y^{\prime}\right) \in \mathbb{R}^{+} \times \partial \omega} \int_{\mathbb{R}^{+} \times \partial \omega}\left|p\left(t / s, x^{\prime}, y^{\prime}\right)\right| \frac{d t}{t} d S_{n-2}\left(x^{\prime}\right)<C .
$$

A standard lemma (the generalized Young's inequality [10]) then gives the result.

Next, we want to extend the definition of the operator valued convolution kernel of an operator in $\mathcal{R}_{-\infty}$ to operators in $\mathcal{R}_{m}$. To do this, we choose an approximate unit $\phi_{j} \in \mathcal{R}_{-\infty}$. Let $P \in \Psi^{m}\left(\mathbb{R}^{+} \times \partial \omega\right)^{\mathbb{R}^{+}}$. Define $P_{j}=C_{\phi_{j}} P$. Hence $P_{j} \in \mathcal{R}_{-\infty}$. Denote the kernel of $P_{j}$ by $k_{P_{j}}$. Then the convolution kernel of $P$ is defined to be $k_{p}=\lim _{j \rightarrow \infty} k_{P_{j}}$. See $[5,27]$.

We turn our attention to studying the mapping property for $P \in \mathcal{R}_{k}$. Denote by $g$ the cylindrical metric on $\mathbb{R}^{+} \times \partial \omega$, that is, $g=\left(r^{-1} d r\right)^{2}+\left(d x^{\prime}\right)^{2}$, where $\left(d x^{\prime}\right)^{2}$ is the metric on $\partial \omega$ induced from the Euclidean metric on $\mathbb{R}^{n}$. Let $L^{2}\left(\mathbb{R}^{+} \times \partial \omega, g\right)$ be the $L^{2}$ space defined by the volume form determined by the metric $g$ and let $H^{s}\left(\mathbb{R}^{+} \times \partial \omega, g\right)$ be the Sobolev spaces defined by the same metric. Let $\Delta_{g}:=$ $\left(r \partial_{r}\right)^{2}+\Delta_{\partial \omega}$ be the Laplacian associated to the metric $g$. Then $H^{s}\left(\mathbb{R}^{+} \times \partial \omega, g\right)$ is obtained as the domain of the powers of $\Delta_{g}$.

Theorem 2.10. Let $P \in \mathcal{R}_{-\infty}$. Then for all $k, l \in \mathbb{Z}$, it defines a continuous map

$$
P: H^{-2 k}\left(\mathbb{R}^{+} \times \partial \omega, g\right) \rightarrow H^{2 l}\left(\mathbb{R}^{+} \times \partial \omega, g\right) .
$$

Proof. Let $Q=\left(I-\Delta_{g}\right)^{l} P\left(I-\Delta_{g}\right)^{k}$. By Proposition 2.5 and Definition 2.6, we have $Q \in \mathcal{R}_{-\infty}$. Then the desired result follows from Lemma 2.9 and the fact that

$$
\left(I-\Delta_{\partial \Omega}\right)^{k}: H^{2 k}\left(\mathbb{R}^{+} \times \partial \omega, g\right) \rightarrow L^{2}\left(\mathbb{R}^{+} \times \partial \omega, g\right)
$$

is an isomorphism.

Theorem 2.11. If $P \in \Psi_{\text {prop }}^{k}\left(\mathbb{R}^{+} \times \partial \omega\right)^{\mathbb{R}^{+}}$, then for all $m \in \mathbb{Z}$, we have that $P$ defines a bounded operator

$$
P: H^{m}\left(\mathbb{R}^{+} \times \partial \omega, g\right) \rightarrow H^{m-k}\left(\mathbb{R}^{+} \times \partial \omega, g\right) .
$$

Proof. First of all, suppose that $P \in \Psi_{\text {prop }}^{-a}\left(\mathbb{R}^{+} \times \partial \omega\right)^{\mathbb{R}^{+}}$with $a>0$ and $f \in$ $L^{2}\left(\mathbb{R}^{+} \times \partial \omega\right)$. Since $\|P f\|_{L^{2}\left(\mathbb{R}^{+} \times \partial \omega\right)}^{2}=\left(P^{*} P f, f\right)_{L^{2}\left(\mathbb{R}^{+} \times \partial \omega\right)}$, it is enough to show that $Q:=P^{*} P$ is bounded on $L^{2}\left(\mathbb{R}^{+} \times \partial \omega\right)$. But for $i$ large enough, by Lemma 2.9, $Q^{i} \in \Psi_{\text {prop }}^{-2 i}\left(\mathbb{R}^{+} \times \partial \omega\right)$ is bounded on $L^{2}\left(\mathbb{R}^{+} \times \partial \omega\right)$. Hence, if $P \in \Psi_{\text {prop }}^{-a}\left(\mathbb{R}^{+} \times \partial \omega\right)^{\mathbb{R}^{+}}$, then $P$ is bounded on $L^{2}\left(\mathbb{R}^{+} \times \partial \omega\right)$.

Then we can use the standard Hörmander trick (see for example, [36]) to show that if $P \in \Psi_{\text {prop }}^{0}\left(\mathbb{R}^{+} \times \partial \omega\right)^{\mathbb{R}^{+}}$, then $P$ defines a bounded operator

$$
P: L^{2}\left(\mathbb{R}^{+} \times \partial \omega, g\right) \rightarrow L^{2}\left(\mathbb{R}^{+} \times \partial \omega, g\right) .
$$

This completes the proof.

We therefore obtain

Corollary 2.12. Let $P \in \mathcal{R}_{k}$, then for all $m \in \mathbb{Z}$, we have that $P$ defines a bounded operator

$$
P: H^{m}\left(\mathbb{R}^{+} \times \partial \omega, g\right) \rightarrow H^{m-k}\left(\mathbb{R}^{+} \times \partial \omega, g\right) .
$$




\section{Convolution and the Mellin Transform}

A standard tool in the study of domains with conical points is the Mellin Transform $[5,8,15,25]$. Recall that the Mellin transform $\mathcal{M}^{\sharp} f$ of $f \in \mathcal{C}_{c}\left(\mathbb{R}^{+}\right)$is given by

$$
\mathcal{M}^{\sharp} f(t)=\int_{0}^{\infty} r^{-i t} f(r) \frac{d r}{r} .
$$

We extend this definition to $f \in \mathcal{C}_{c}(\Omega)$ by

$$
\mathcal{M}^{\sharp} f\left(t, x^{\prime}\right)=\int_{0}^{\infty} r^{-i t} f\left(r x^{\prime}\right) \frac{d r}{r} .
$$

A similar formula defines $\mathcal{M}^{\sharp} f\left(t, x^{\prime}\right)$ if $f \in \mathcal{C}_{c}(\partial \Omega)$. As for the Fourier transform, the Mellin transform extends to $L^{2}\left(\Omega, d \mu_{n}\right)$ and to $L^{2}\left(\partial \Omega, d \mu_{n-1}\right)$, where $\mu_{n}$ and $\mu_{n-1}$ are the measures defined in Equation (6).

Proposition 3.1. The map

$$
(2 \pi)^{-1 / 2} \mathcal{M}^{\sharp}: L^{2}\left(\Omega, d \mu_{n}\left(x^{\prime}\right)\right) \rightarrow L^{2}\left(\mathbb{R} \times \omega, d t d S_{n-1}\left(x^{\prime}\right)\right)
$$

is unitary.

Proof. The Plancherel formula for Fourier transform implies the result since the Mellin transform is the composition of

$$
L^{2}\left(\Omega, d \mu_{n}\right) \ni f\left(r x^{\prime}\right) \rightarrow f\left(e^{t} x^{\prime}\right) \in L^{2}\left(\mathbb{R} \times \omega, d t d S_{n-1}\left(x^{\prime}\right)\right)
$$

with the Fourier transform in the $t$ variable.

Definition 3.2. Let $p$ be the convolution kernel of a smoothing Mellin convolution operator $P$ on $\Omega$ or on $\partial \Omega$. The Mellin transform $\mathcal{M}^{\sharp} p$ of $p$ is defined by

$$
\mathcal{M}^{\sharp} p\left(t, x^{\prime}, y^{\prime}\right)=q\left(t, x^{\prime}, y^{\prime}\right)=\int_{0}^{\infty} s^{-i t} p\left(s, x^{\prime}, y^{\prime}\right) \frac{d s}{s} .
$$

The Mellin transform $\mathcal{M}^{\sharp} P(t)$ of $P$ is defined to be integral kernel operator with kernel $\mathcal{M}^{\sharp} p\left(t, x^{\prime}, y^{\prime}\right)$. This definition extends to $P \in \mathcal{R}_{k}$ by allowing $p$ to be a distribution with singular support at 1 .

We shall need the following.

Example 3.3. We have

$$
\mathcal{M}^{\sharp}\left(r \partial_{r}\right)(t)=\left.\left(r \partial_{r}\right)^{*} r^{-i t}\right|_{r=1}=-\left.\left(r \partial_{r}\right) r^{-i t}\right|_{r=1}=i t,
$$

where $\left(r \partial_{r}\right)^{*}$ is the adjoint with respect to the measure $\mu_{n}$ or $\mu_{n-1}$. On the other hand, if $P$ is a differential operator that acts only on the $x^{\prime}$ variable, then $\mathcal{M}^{\sharp}(P)=$ $P$.

Remark 3.4. Let us denote by $\Im(z)=b$ the imaginary part of a complex number $z=a+b i$. If $P \in \mathcal{R}_{-\infty}$, then $\left(\mathcal{M}^{\sharp} P\right)(t)$ extends to a holomorphic function in $t \in\{|\Im(t)|<\epsilon\}$, with $\epsilon$ as in the Definition 2.6. On the other hand, if $P \in$ $\Psi_{\text {prop }}^{k}\left(\mathbb{R}^{+} \times \partial \omega\right)^{\mathbb{R}^{+}}$, then $\left(\mathcal{M}^{\sharp} P\right)(t)$ is defined for all $t \in \mathbb{C}$.

Let us recall the following standard properties of the Mellin transform. 
Proposition 3.5. Suppose $P \in \mathcal{R}_{k}$ is a Mellin convolution operator on $\partial \Omega$ with operator-valued convolution kernel $p$. Then $\mathcal{M}^{\sharp} P(t) \in \Psi^{k}(\partial \omega)$ and for any $u \in$ $\mathcal{C}_{c}(\partial \Omega)$, we have

$$
\mathcal{M}^{\sharp}(p * u)(t)=\mathcal{M}^{\sharp}(P u)(t)=\mathcal{M}^{\sharp} P(t) \mathcal{M}^{\sharp} u(t) .
$$

The same is true if $P$ is a smoothing Mellin convolution operator on $\Omega$.

Proof. We have that $\mathcal{M}^{\sharp} P(t) \in \Psi^{k}(\partial \omega)$ by [5, 27]. Let $v\left(t, x^{\prime}\right)=(P u)\left(t, x^{\prime}\right)$. Let us assume first that $u \in \mathcal{C}_{c}(\partial \Omega)$ and $P \in \mathcal{R}_{-\infty}$. Then we calculate using Fubini's theorem to interchange the order of integration.

$$
\begin{aligned}
\mathcal{M}^{\sharp} v\left(t, x^{\prime}\right) & =\int_{0}^{\infty} r^{-i t} v\left(r, x^{\prime}\right) \frac{d r}{r} \\
& =\int_{0}^{\infty} r^{-i t}\left(\int_{0}^{\infty} \int_{\omega} p\left(r / s, x^{\prime}, y^{\prime}\right) u\left(s, y^{\prime}\right) d S_{n-1}\left(y^{\prime}\right) \frac{d s}{s}\right) \frac{d r}{r} \\
& =\int_{\omega}\left(\int_{0}^{\infty} \int_{0}^{\infty}(r / s)^{-i t} p\left(r / s, x^{\prime}, y^{\prime}\right) s^{-i t} u\left(s, y^{\prime}\right) \frac{d r}{r} \frac{d s}{s}\right) d S_{n-1}\left(y^{\prime}\right) \\
& =\int_{\omega}\left(\int_{0}^{\infty} z^{-i t} p\left(z, x^{\prime}, y^{\prime}\right) \frac{d z}{z}\right)\left(\int_{0}^{\infty} s^{-i t} u\left(s, y^{\prime}\right) \frac{d s}{s}\right) d S_{n-1}\left(y^{\prime}\right) \\
& =\int_{\omega} \mathcal{M}^{\sharp} p\left(t, x^{\prime}, y^{\prime}\right) \mathcal{M}^{\sharp} u\left(t, y^{\prime}\right) d S_{n-1}\left(y^{\prime}\right)=\left(\mathcal{M}^{\sharp} P \mathcal{M}^{\sharp} u\right)\left(t, x^{\prime}\right) .
\end{aligned}
$$

The general case $P \in \mathcal{R}_{k}$ follows by considering an approximate unit $\phi_{j} \in \mathcal{R}_{-\infty}$, that is a sequence such that $\phi_{j} * f \rightarrow f$. This completes the proof.

Let us denote by $M_{f}$ the multiplication operator by $f$.

Proposition 3.6. Let $P \in \mathcal{R}_{k}$ be a Mellin convolution operator on $\partial \Omega$ with operatorvalued convolution kernel $p\left(r, x^{\prime}, y^{\prime}\right)$. Then $Q:=M_{r^{a}} P M_{r^{-a}}$ is still a convolution operator with kernel $k_{Q}\left(r, x^{\prime}, y^{\prime}\right)=r^{a} p\left(r, x^{\prime}, y^{\prime}\right)$. The same is true if $P$ is a smoothing Mellin convolution operator on $\Omega$.

Proof. If $u \in \mathcal{C}_{c}(\partial \Omega)$, we have

$$
\begin{aligned}
\left(M_{r^{a}} P M_{r^{-a}} u\right)\left(r, x^{\prime}\right) & =\int_{\omega} r^{a} \int_{0}^{\infty} p\left(r / s, x^{\prime}, y^{\prime}\right) s^{-a} u\left(s, y^{\prime}\right) \frac{d s}{s} d S_{n-1}\left(y^{\prime}\right) \\
& =\int_{\omega} \int_{0}^{\infty}(r / s)^{a} p\left(r / s, x^{\prime}, y^{\prime}\right) u\left(s, y^{\prime}\right) \frac{d s}{s} d S_{n-1}\left(y^{\prime}\right),
\end{aligned}
$$

where the integrals represent pairings of distributions and functions. We have thus completed the proof.

This gives the following.

Corollary 3.7. Let $P \in \Psi_{\text {prop }}^{k}\left(\mathbb{R}^{+} \times \partial \omega\right)^{\mathbb{R}^{+}}$. Then

$$
r^{z} \operatorname{Pr}^{-z} \in \Psi_{\text {prop }}^{k}\left(\mathbb{R}^{+} \times \partial \omega\right)^{\mathbb{R}^{+}}
$$

for all $z \in \mathbb{C}$. Also, if $P \in \mathcal{R}_{-\infty}$, then $r^{z} \operatorname{Pr}^{-z} \in \mathcal{R}_{-\infty}$ if $|\Im(z)|<\epsilon$, where $\epsilon$ is as in Definition 2.6.

The above corollary allows us to extend the Mellin transform to the class of operators $P$ such that $r^{a} \mathrm{Pr}^{-a} \in \mathcal{R}_{k}$ for some $a$ and $k$. The Mellin transform of $P$ will then be defined and holomorphic in a set containing $|\Im(z-a)|<\epsilon$ form 
some small $\epsilon>0$. Also, we shall agree that the Mellin transform is extended holomorphically to the largest set of the form $\{a<\Im(z)<b\}$.

The Lebesgue measure on $\mathbb{R}^{n}$ is given in spherical coordinates by

$$
d x=r^{n-1} d r d S_{n-1}\left(x^{\prime}\right),
$$

where $d S_{n-1}\left(x^{\prime}\right)$ is the surface measure on the unit sphere in $\mathbb{R}^{n}$. Consider the Hilbert spaces $L^{2}(\Omega, d x)$ and $L^{2}\left(\Omega, d \mu_{n}\left(x^{\prime}\right)\right)$. Then the map

$$
M_{r^{\frac{n}{2}}}: L^{2}(\Omega, d x) \rightarrow L^{2}\left(\Omega, d \mu_{n}\left(x^{\prime}\right)\right)
$$

is unitary.

Definition 3.8. We define the shifted Mellin transform on $\Omega$

$$
\mathcal{M}: L^{2}(\Omega, d x) \rightarrow L^{2}\left(\mathbb{R} \times \omega, d t d S_{n-1}\left(x^{\prime}\right)\right)
$$

by the formula

$$
\mathcal{M} u=\mathcal{M}^{\sharp}\left(M_{r^{\frac{n}{2}}} u\right),
$$

so $\mathcal{M} u(t)=\mathcal{M}^{\sharp} u(t+i n / 2)$.

Since the measure on $\partial \Omega$ is $r^{n-2} d r d S_{n-2}\left(x^{\prime}\right)$, for $\partial \Omega$ we adapt the above definition as follows.

Definition 3.9. We define the shifted Mellin transform on the boundary by $\partial \Omega$,

$$
\mathcal{M}_{\partial \Omega}: L^{2}\left(\partial \Omega, r^{n-2} d r d S_{n-2}\left(x^{\prime}\right)\right) \rightarrow L^{2}\left(\mathbb{R} \times \partial \omega, d t d S_{n-2}\left(x^{\prime}\right)\right),
$$

by the formula

$$
\left(\mathcal{M}_{\partial \Omega} f\right)\left(t, x^{\prime}\right)=\mathcal{M}^{\sharp}\left(M_{r^{\frac{n-1}{2}}} f\right),
$$

so $\mathcal{M}_{\partial \Omega} f(t)=\mathcal{M}^{\sharp} f(t+i(n-1) / 2)$.

The shift in Definition 3.9 is necessary to make $\mathcal{M}_{\partial \Omega}$ unitary up to a multiple. We will drop $\partial \Omega$ if no confusion can arise.

\section{Lemma 3.10.}

(i) Let $P$ be a smoothing Mellin convolution operator on $\Omega$ with operator-valued convolution kernel $p\left(r, x^{\prime}, y^{\prime}\right)$. Then for any $u \in \mathcal{C}_{c}(\Omega)$, we have

$$
\mathcal{M}(p * u)(t)=\mathcal{M}(P u)(t)=\mathcal{M} P(t) \mathcal{M} u(t)
$$

and $\mathcal{M}(P)=\mathcal{M}^{\sharp}\left(M_{r^{\frac{n}{2}}} P M_{r^{-\frac{n}{2}}}\right)$.

(ii) Let $P \in \mathcal{R}_{k}$ be a Mellin convolution operator on $\partial \Omega$ with operator-valued convolution kernel $p\left(r, x^{\prime}, y^{\prime}\right)$. Then for any $f \in \mathcal{C}_{c}(\partial \Omega)$, we have

$$
\begin{aligned}
\mathcal{M}(p * f)(t)=\mathcal{M}(P f)(t)=\mathcal{M} P(t) \mathcal{M} f(t) \\
\text { and } \mathcal{M}(P)=\mathcal{M}^{\sharp}\left(M_{r^{\frac{n-1}{2}}} P M_{r^{-\frac{n-1}{2}}}\right) .
\end{aligned}
$$

Proof. The results immediately follow from Proposition 3.5 and Proposition 3.6

Definition 3.11. We shall denote by $\mathcal{A}$ the completion of $\mathcal{R}_{-1}$ in the norm of bounded operators acting on $L^{2}\left(\partial \Omega, d \mu_{n-1}\right)$.

Denote by $\mathcal{A}^{+}$the unitalization of $\mathcal{A}$ and by $\mathcal{C}_{0}\left(\mathbb{R}, \mathcal{K}\left(L^{2}(\partial \omega)\right)\right)$ the $C^{*}$-algebra of all continuous functions from $\mathbb{R}$ to $\mathcal{K}\left(L^{2}(\partial \omega)\right)$ that vanish at infinity.

Theorem 3.12. The Mellin transform $\mathcal{M}^{\sharp}$ induces an isomorphism from $\mathcal{A}$ to the $C^{*}$-algebra $\mathcal{C}_{0}\left(\mathbb{R}, \mathcal{K}\left(L^{2}(\partial \omega)\right)\right)$. Moreover, $\lambda+P$ is invertible in $\mathcal{A}^{+}$if, and only if $\lambda+\left(\mathcal{M}^{\sharp} P\right)(t)$ is invertible in $\mathcal{L}\left(L^{2}(\partial \omega)\right)$ for all $t \in \mathbb{R}$. 
We need some preparations to prove this theorem.

Lemma 3.13. Let $n \in \mathbb{N}_{0} \cup\{\infty\}$ be arbitrary and $\mathcal{B}$ be a Banach algebra. Suppose that $\kappa \in \mathcal{C}^{n}(\mathbb{R}, \mathcal{B})$ fulfills $\kappa(t)^{-1} \in \mathcal{B}$ for all $t \in \mathbb{R}$. Then $\kappa^{-1} \in \mathcal{C}^{n}(\mathbb{R}, \mathcal{B})$ also. In particular, for $\kappa \in \mathcal{C}(\mathbb{R}, \mathcal{B})$, we have

$$
\kappa^{-1} \in \mathcal{C}(\mathbb{R}, \mathcal{B}) \text { exists } \Longleftrightarrow \kappa(t)^{-1} \in \mathcal{B} \text { exists for all } t \in \mathbb{R} \text {. }
$$

Proof. First of all, recall that since $\mathcal{B}$ is a Banach algebra, the group of its invertible elements $\mathcal{B}^{-1}$ is open and the inversion $\mathcal{B}^{-1} \ni a \longmapsto a^{-1}$ is infinitely many times differentiable. Let us first treat the case $n=0$ : It is clear, that the existence of $\kappa^{-1} \in \mathcal{C}(\mathbb{R}, \mathcal{B})$ implies that $\kappa(t)$ is invertible for all $t \in \mathbb{R}$. Conversely, assume that $\kappa(t)^{-1} \in \mathcal{B}$ exist for all $t \in \mathbb{R}$. We define $\kappa^{-1}(t):=\kappa(t)^{-1}$. Then we have to check that $\kappa^{-1} \in \mathcal{C}(\mathbb{R}, \mathcal{B})$ holds. Let $t_{0} \in \mathbb{R}$ be arbitrary:

$$
\kappa\left(t_{0}\right)^{-1}-\kappa(t)^{-1}=\kappa\left(t_{0}\right)^{-1}\left(\kappa(t)-\kappa\left(t_{0}\right)\right) \kappa^{-1}(t)
$$

which gives $\kappa^{-1} \in \mathcal{C}(\mathbb{R}, \mathcal{B})$ after passing to the limit $t \rightarrow t_{0}$, since $\mathcal{B}$ has an open group of invertible elements and the inversion is continuous.

Now, if $n=1$, we use

$$
\frac{\kappa(t)^{-1}-\kappa\left(t_{0}\right)^{-1}}{t-t_{0}}=-\kappa\left(t_{0}\right)^{-1} \frac{\kappa\left(t_{0}\right)-\kappa(t)}{t_{0}-t} \kappa(t)^{-1},
$$

to see the differentiability of $\kappa^{-1}$. An iteration of this argument yields then the assertion.

Proposition 3.14. We have the following:

(i) $\mathcal{R}_{-\infty} \subset \mathcal{A}$;

(ii) If $P$ is a Mellin convolution operator and $P \in \Psi_{\text {prop }}^{-1}\left(\mathbb{R}^{+} \times \partial \omega\right)^{\mathbb{R}^{+}}$, then $\mathcal{M}^{\sharp} P \in$ $\mathcal{C}_{0}\left(\mathbb{R}, \mathcal{K}\left(L^{2}(\partial \omega)\right)\right)$.

Proof. The first statement is clear. By Theorem 8.8 in [10], if $P \in \Psi_{\text {prop }}^{-n}\left(\mathbb{R}^{+} \times\right.$ $\partial \omega)^{\mathbb{R}^{+}}$, then $p\left(r, x^{\prime}, y^{\prime}\right)$ is continuous for all variables. We notice that if $P \in$ $\Psi_{\text {prop }}^{-n}\left(\mathbb{R}^{+} \times \partial \omega\right)^{\mathbb{R}^{+}}$, by Lemma 2.9 , there exists a constant $C$ such that for all $t \in \mathbb{R}$

$$
\left\|\left(\mathcal{M}^{\sharp} P\right)(t)\right\| \leqslant C .
$$

Now, if $P \in \Psi_{\text {prop }}^{-n-1}\left(\mathbb{R}^{+} \times \partial \omega\right)^{\mathbb{R}^{+}}$, then $\left(1+r \partial_{r}\right) P \in \Psi_{\text {prop }}^{-n}\left(\mathbb{R}^{+} \times \partial \omega\right)^{\mathbb{R}^{+}}$. Also we have

$$
\mathcal{M}^{\sharp}\left(\left(1+r \partial_{r}\right) P\right)(t)=(1+i t)\left(\mathcal{M}^{\sharp} P\right)(t) .
$$

So we obtain

$$
\left\|\left(\mathcal{M}^{\sharp} P\right)(t)\right\| \leqslant C(1+|t|)^{-1} \rightarrow 0 \quad \text { as }|t| \rightarrow \infty .
$$

This implies $\mathcal{M}^{\sharp} P \in \mathcal{C}_{0}\left(\mathbb{R}, \mathcal{K}\left(L^{2}(\partial \omega)\right)\right)$.

We then use Hörmander's trick to complete the proof of the second statement. Suppose $P \in \Psi_{\text {prop }}^{-1}\left(\mathbb{R}^{+} \times \partial \omega\right)^{\mathbb{R}^{+}}$and $f \in L^{2}\left(\mathbb{R}^{+} \times \partial \omega\right)$. Since $\|P f\|_{L^{2}\left(\mathbb{R}^{+} \times \partial \omega\right)}^{2}=$ $\left(P^{*} P f, f\right)_{L^{2}\left(\mathbb{R}^{+} \times \partial \omega\right)}$, it suffices to prove that some power of $Q:=P^{*} P$ is in $\Psi_{\text {prop }}^{-n-2}\left(\mathbb{R}^{+} \times \partial \omega\right)^{\mathbb{R}^{+}}$. But $Q^{j} \in \Psi_{\text {prop }}^{-2 j}\left(\mathbb{R}^{+} \times \partial \omega\right)$, so for $j$ large enough, this follows from the above discussion. 
We are ready now to complete the proof of Theorem 3.12.

Proof of Theorem 3.12. By Proposition 3.14, we have if $P \in \mathcal{R}_{-1}$, then $\mathcal{M}^{\sharp} P \in$ $\mathcal{C}_{0}\left(\mathbb{R}, \mathcal{K}\left(L^{2}(\partial \omega)\right)\right)$. Since $\mathcal{C}_{0}\left(\mathbb{R}, \mathcal{K}\left(L^{2}(\partial \omega)\right)\right) \cong \mathcal{C}_{0}(\mathbb{R}) \otimes \mathcal{K}\left(L^{2}(\partial \omega)\right)$ and the set

$$
\operatorname{span}\left\{(f T)(t):=f(t) T \mid f \in \mathcal{C}_{0}(\mathbb{R}), T \in \mathcal{K}\left(L^{2}(\partial \omega)\right)\right\}
$$

is dense in $\mathcal{C}_{0}(\mathbb{R}) \otimes \mathcal{K}\left(L^{2}(\partial \omega)\right)$, the image of $\mathcal{R}_{-1}$ under $\mathcal{M}^{\sharp}$ is dense in $\mathcal{C}_{0}\left(\mathbb{R}, \mathcal{K}\left(L^{2}(\partial \omega)\right)\right)$. This gives surjectivity. To prove injectivity, we notice that $\mathcal{A}=\mathcal{K}\left(L^{2}(\partial \omega)\right) \rtimes_{r} \mathbb{R}$ (reduced crossed-product), where $\mathbb{R}$ acts on $\mathcal{K}\left(L^{2}(\partial \omega)\right)$ trivially. Since $\mathbb{R}$ is commutative and its action is trivial, we have $\mathcal{K}\left(L^{2}(\partial \omega)\right) \rtimes_{r} \mathbb{R} \cong \mathcal{K}\left(L^{2}(\partial \omega)\right) \otimes \mathcal{C}_{0}(\mathbb{R})$. Hence the Mellin transform $\mathcal{M}^{\sharp}$ is an isomorphism. Let us also notice by the results of [21], the algebra $\mathcal{R}_{-1}$ is closed under holomorphic functional calculus in $\mathcal{A}$. Similarly, $\mathcal{R}_{0}$ is also closed under holomorphic functional calculus.

The second part follows from Lemma 3.13.

\section{The double layer potential operator $K$ on Straight Cones}

Let $\omega \subset S^{n-1}(n \geqslant 3)$ be an open subset with smooth boundary. We allow $\omega$ to be disconnected. Denote by $\Omega=\mathbb{R}^{+} \omega$ the cone with base $\omega$, as before (Equation (5)). We denote by $d S_{n-1}(x)$ be the surface measure with respect to $\partial \Omega$. In these generalized spherical coordinates, the Laplacian $\Delta=\sum_{j} \partial_{j}^{2}$ on $\Omega$ is given by

$$
\Delta=r^{-2}\left(\left(r \partial_{r}\right)^{2}+(n-2) r \partial_{r}+\Delta_{S^{n-1}}\right),
$$

where $\Delta_{S^{n-1}}$ is the Laplacian on $S^{n-1}$. Let

$$
E(x, y)=\frac{1}{(2-n) \omega_{n}} \frac{1}{|x-y|^{n-2}}=\frac{c_{n}}{|x-y|^{n-2}}
$$

be the standard fundamental solution for $\Delta$ (so $\omega_{n}$ is the area of the unit sphere in $\left.\mathbb{R}^{n}\right)$. We shall denote $K=K(\Delta ; E ; \Omega), S=S(\Delta ; E ; \Omega)$.

Let $\nu$ denote the outward unit normal vector. A convenient way of writing the operators $K$ and $S$ for the cone takes into account the dilation invariance of the cone. Let $x=r x^{\prime}, y=s y^{\prime}$, and denote by $d S_{n-2}\left(y^{\prime}\right)$ the measure on $\partial \omega$ and $\nu(y)$ the unit outer normal vector to $\partial \Omega$ at the point $y \in \partial \Omega$. Then the double layer potential operator $K$ can be written as

$$
\begin{aligned}
K g(x) & =\int_{\partial \Omega} \frac{\partial E}{\partial_{\nu_{y}}}(x, y) g(y) d S_{n-1}(y) \\
& =-\int_{\partial \Omega} \frac{(x-y) \cdot \nu(y)}{\omega_{n}|x-y|^{n}} g(y) d S_{n-1}(y) \\
& =-\frac{1}{\omega_{n}} \int_{0}^{\infty} \int_{\partial \omega} \frac{\left(r x^{\prime}-s y^{\prime}\right) \cdot \nu\left(y^{\prime}\right)}{\left|r x^{\prime}-s y^{\prime}\right|^{n}} g\left(s y^{\prime}\right) s^{n-2} d S_{n-2}\left(y^{\prime}\right) d s \\
& =-\frac{1}{\omega_{n}} \int_{0}^{\infty} \int_{\partial \omega} \frac{\frac{r}{s} x^{\prime} \cdot \nu\left(y^{\prime}\right)}{\left|\frac{r}{s} x^{\prime}-y^{\prime}\right|^{n}} g\left(s y^{\prime}\right) d S_{n-2}\left(y^{\prime}\right) \frac{d s}{s} .
\end{aligned}
$$

Thus Equation (10) shows that $K$ is a Mellin convolution operator with the operator-valued convolution kernel

$$
k\left(r, x^{\prime}, y^{\prime}\right):=-\frac{1}{\omega_{n}} \frac{r x^{\prime} \cdot \nu\left(y^{\prime}\right)}{\left|r x^{\prime}-y^{\prime}\right|^{n}}=-\frac{1}{\omega_{n}} \frac{r x^{\prime} \cdot \nu\left(y^{\prime}\right)}{\left(r^{2}-2 r x^{\prime} \cdot y^{\prime}+1\right)^{n / 2}} .
$$


To obtain similar results for the single layer potential operator $S$, we shall need the operator $\Phi: \mathcal{C}_{c}^{\infty}(\Omega) \rightarrow L_{\text {loc }}^{2}(\Omega)$ defined by

$$
\begin{aligned}
(\Phi u)(x) & =\int_{\Omega} \frac{c_{n}}{|x-y|^{n-2}} u(y) d y \\
& =\int_{0}^{\infty} \int_{\omega} \frac{c_{n}}{\left|r x^{\prime}-s y^{\prime}\right|^{n-2}} u\left(s y^{\prime}\right) s^{n-1} d S_{n-1}\left(y^{\prime}\right) d s \\
& =\int_{0}^{\infty} \int_{\omega} \frac{c_{n} s^{2}}{\left|\frac{r}{s} x^{\prime}-y^{\prime}\right|^{n-2}} u\left(s y^{\prime}\right) d S_{n-1}\left(y^{\prime}\right) \frac{d s}{s}
\end{aligned}
$$

where $x=r x^{\prime}, y=s y^{\prime}$ and $d S_{n-1}\left(y^{\prime}\right)$ denotes the surface measure on the unit sphere in $\mathbb{R}^{n}$.

Let $\Phi_{0}=M_{r^{-1}} \Phi M_{r^{-1}}$. Then for $u \in \mathcal{C}_{c}(\Omega)$, we have

$$
\Phi_{0} u(x)=\int_{0}^{\infty} \int_{\omega} \frac{c_{n}}{\left(\frac{r}{s}\right)\left|\frac{r}{s} x^{\prime}-y^{\prime}\right|^{n-2}} u\left(s y^{\prime}\right) d S_{n-1}\left(y^{\prime}\right) \frac{d s}{s} .
$$

From Equation (13), we see that $\Phi_{0}$ is a Mellin convolution operator with kernel

$$
k_{\Phi_{0}}\left(r, x^{\prime}, y^{\prime}\right)=\phi_{0}\left(r, x^{\prime}, y^{\prime}\right)=\frac{c_{n}}{r\left|r x^{\prime}-y^{\prime}\right|^{n-2}} .
$$

Lemma 4.1. For any $t \in \mathbb{R}^{+}$, we have

(i) $K \alpha_{t}=\alpha_{t} K$;

(ii) $\Phi_{0} \alpha_{t}=\alpha_{t} \Phi_{0}$;

(iii) $\left(r \partial_{r}\right)^{k} \alpha_{t}=\alpha_{t}\left(r \partial_{r}\right)^{k}$, where $r$ is the radial variable.

Proof. Since $K$ and $\Phi_{0}$ are both Mellin convolution operators, the conclusions of (i) and (ii) follow from Lemma 2.3. To prove (iii), it is enough to notice that $r \partial_{r}=\left.\alpha_{t}^{\prime}\right|_{t=0}$

Proposition 4.2. For $u \in \mathcal{C}_{c}^{1}(\Omega)$, we have

$$
\mathcal{M}\left(r \partial_{r} u\right)(t)=\left(i t-\frac{n}{2}\right)(\mathcal{M} u)(t) .
$$

Proof. This follows from Example 3.3 and Lemma 3.5. Indeed, we have $\mathcal{M}\left(r \partial_{r}\right)=$ $\mathcal{M}^{\sharp}\left(r^{n / 2}\left(r \partial_{r}\right) r^{-n / 2}\right)=\mathcal{M}^{\sharp}\left(r \partial_{r}-n / 2\right)=i t-n / 2$.

We shall need the range for which $\mathcal{M}\left(\Phi_{0}\right)$ is defined.

Let $\operatorname{Diff}^{2}(\omega)$ denote the set of differential operators of order two on $\omega$, and also let $\Delta_{0}=M_{r} \Delta M_{r}$.

Lemma 4.3. Let $\widetilde{\Delta}_{0}:=M_{r^{\frac{n}{2}}}\left(\Delta_{0}\right) M_{r^{-\frac{n}{2}}}$ and $\widetilde{\Phi}_{0}=M_{r^{\frac{n}{2}}} \Phi_{0} M_{r^{-\frac{n}{2}}}$.

(i) $\mathcal{M}\left(\Delta_{0}\right)(t)=\mathcal{M}^{\sharp} \widetilde{\Delta}_{0}(t) \in \operatorname{Diff}^{2}(\omega)$;

(ii) $\mathcal{M} \Phi_{0}=\mathcal{M}^{\sharp} \widetilde{\Phi}_{0}$;

(iii) The operator $\widetilde{\Phi}_{0}$ has Mellin convolution kernel

$$
\widetilde{\phi}_{0}\left(r, x^{\prime}, y^{\prime}\right)=\frac{c_{n}}{\left|r^{\frac{1}{2}} x^{\prime}-r^{-\frac{1}{2}} y^{\prime}\right|^{n-2}},
$$

where $x^{\prime}, y^{\prime} \in \omega$. 
Proof. By Lemma 3.10, the first two statements are clear from the definitions. We need to check that $\widetilde{\Phi}_{0}$ has convolution kernel $\widetilde{\phi}_{0}$. Indeed, by Proposition 3.6, the operator $\widetilde{\Phi}_{0}$ has Mellin convolution kernel

$$
\widetilde{\phi}_{0}\left(r, x^{\prime}, y^{\prime}\right)=r^{\frac{n}{2}} \phi_{0}=\frac{c_{n} r^{\frac{n}{2}}}{r\left|r x^{\prime}-y^{\prime}\right|^{n-2}}=\frac{c_{n}}{\left|r^{\frac{1}{2}} x^{\prime}-r^{-\frac{1}{2}} y^{\prime}\right|^{n-2}} .
$$

The proof is complete.

We can now prove the following.

Lemma 4.4. For $u \in \mathcal{C}_{c}^{\infty}(\Omega)$, we have

$$
\mathcal{M}\left(\Phi_{0} u\right)(t)=\mathcal{M} \Phi_{0}(t) \mathcal{M} u(t)
$$

provided that $\Im(t) \in\left(-\frac{n-2}{2}, \frac{n-2}{2}\right)$.

Proof. Equation (15) follows right away from Lemma 3.10, whenever both sides are defined. To check the range for which $\mathcal{M} \Phi_{0}$ is defined, we use Lemma 4.3 (ii) and (iii) that identifies the convolution kernel $\tilde{\phi}_{0}$ of $\widetilde{\Phi}_{0}$. Then we notice that for $\alpha \in\left(-\frac{n-2}{2}, \frac{n-2}{2}\right)$

$$
\left|r^{\alpha} \tilde{\phi}_{0}\left(r, x^{\prime}, y^{\prime}\right)\right|=\frac{c_{n} r^{\alpha}}{\left|r^{\frac{1}{2}} x^{\prime}-r^{-\frac{1}{2}} y^{\prime}\right|^{n-2}} .
$$

decays fast as $r \rightarrow 0$ or $r \rightarrow \infty$.

Denote by $\Delta_{\omega}$ the restriction of $\Delta_{S^{n-1}}$ to $\omega \subset S^{n-1}$. Let $\Phi_{0}=M_{r^{-1}} \Phi M_{r^{-1}}$, as before, and also recall that $\Delta_{0}=M_{r} \Delta M_{r}$.

Proposition 4.5. We have

$$
\mathcal{M}(\Delta \Phi)(t)=\mathcal{M}\left(\Delta_{0} \Phi_{0}\right)(t)=\left(\Delta_{\omega}-t^{2}-\frac{(n-2)^{2}}{4}\right)\left(\mathcal{M} \Phi_{0}\right)(t)=I .
$$

In particular, the function $\mathcal{M} \phi_{0}(t)$ is a fundamental solution for the operator $\Delta_{\omega}-$ $t^{2}-\frac{(n-2)^{2}}{4}$ and domain $\omega$, provided that $\Im(t) \in\left(-\frac{n-2}{2}, \frac{n-2}{2}\right)$.

Proof. First of all, since $\Delta \Phi=I$, we have

$$
\Delta_{0} \Phi_{0}=M_{r} \Delta \Phi M_{r^{-1}}=M_{r} I M_{r^{-1}}=I .
$$

We compute

$$
M_{r} \Delta M_{r}=\left(r \partial_{r}\right)^{2}+n\left(r \partial_{r}\right)+(n-1)+\Delta_{\omega} .
$$

By Lemma 4.1, both $M_{r} \Delta M_{r}$ and $M_{r^{-1}} \Phi M_{r^{-1}}$ are invariant with respect to dilations. Proposition 4.2 and Lemma 4.4 give

$$
\begin{aligned}
\mathcal{M}(\Delta \Phi)(t) & =\mathcal{M}\left(\Delta_{0} \Phi_{0}\right)(t)=\mathcal{M} \Delta_{0}(t) \mathcal{M} \Phi_{0}(t) \\
& =\left(\left(i t-\frac{n}{2}\right)^{2}+n\left(i t-\frac{n}{2}\right)+(n-1)+\Delta_{\omega}\right)\left(\mathcal{M} \Phi_{0}\right)(t) \\
& =\left(\Delta_{\omega}-t^{2}-\frac{(n-2)^{2}}{4}\right) \mathcal{M} \Phi_{0}(t) .
\end{aligned}
$$

On the other hand, since $\Delta \Phi=I$, we get

$$
\mathcal{M}(\Delta \Phi)(t)=I
$$

From the calculation above, we have $\left(\Delta_{\omega}-t^{2}-\frac{(n-2)^{2}}{4}\right) \mathcal{M} \Phi_{0}(t)=I$. Hence, we have completed the proof. 
Corollary 4.6. We have

$$
\mathcal{M}^{\sharp}\left(\widetilde{\Delta} \widetilde{\Phi}_{0}\right)(t)=\left(\Delta_{\omega}-t^{2}-\frac{(n-2)^{2}}{4}\right) \mathcal{M}^{\sharp} \widetilde{\Phi}_{0}(t)=I .
$$

In particular, the function $\mathcal{M}^{\sharp} \widetilde{\phi}_{0}(t)$ is a fundamental solution for the operator $\Delta_{\omega}-t^{2}-\frac{(n-2)^{2}}{4}$, provided that $\Im(t) \in\left(-\frac{n-2}{2}, \frac{n-2}{2}\right)$.

Proof. This follows right away from Proposition 4.5 and Lemma 4.3.

We now turn our attention to the double layer potential operator $K$.

Lemma 4.7. The operator $\widetilde{K}=M_{r^{\frac{n-1}{2}}} K M_{r^{-\frac{n-1}{2}}}$ has convolution kernel

$$
\widetilde{k}\left(r, x^{\prime}, y^{\prime}\right)=-\frac{1}{\omega_{n}} \frac{r^{\frac{1}{2}} x^{\prime} \cdot \nu\left(y^{\prime}\right)}{\left|r^{\frac{1}{2}} x^{\prime}-r^{-\frac{1}{2}} y^{\prime}\right|^{n}} .
$$

Proof. Recall that the kernel $k$ of double layer potential operator $K$ is given in Equation (11). By Proposition 3.6, the operator $\widetilde{K}$ has Mellin convolution kernel

$$
\widetilde{k}\left(r, x^{\prime}, y^{\prime}\right)=r^{\frac{n-1}{2}} k\left(r, x^{\prime}, y^{\prime}\right)=-\frac{1}{\omega_{n}} \frac{r^{\frac{1}{2}} x^{\prime} \cdot \nu\left(y^{\prime}\right)}{\left|r^{\frac{1}{2}} x^{\prime}-r^{-\frac{1}{2}} y^{\prime}\right|^{n}} .
$$

The proof is complete.

\section{The Single Layer Potential Operator $S$ on Straight Cones}

Let us consider now the single layer potential operator $S$. With the notation in the above section, the single layer potential $S$ satisfies

$$
\begin{aligned}
S g(x) & =\int_{\partial \Omega} \frac{c_{n}}{|x-y|^{n-2}} g(y) d S_{n-1}(y) \\
& =\int_{0}^{\infty} \int_{\partial \omega} \frac{c_{n}}{\left|r x^{\prime}-s y^{\prime}\right|^{n-2}} g\left(s y^{\prime}\right) s^{n-2} d S_{n-2}\left(y^{\prime}\right) d s \\
& =\int_{0}^{\infty} \int_{\partial \omega} \frac{c_{n} r}{\frac{r}{s}\left|\frac{r}{s} x^{\prime}-y^{\prime}\right|^{n-2}} g\left(s y^{\prime}\right) d S_{n-2}\left(y^{\prime}\right) \frac{d s}{s},
\end{aligned}
$$

where $x=r x^{\prime}$ and $y=s y^{\prime}$. The operator $S$ is not dilation invariant, but behaves under dilations like $r$. This suggests to introduce $S_{0}:=M_{r^{-\frac{1}{2}}} S M_{r^{-\frac{1}{2}}}$, then, unlike $S, S_{0}$ is a Mellin convolution operator, and its operator-valued convolution kernel is given by

$$
h\left(r, x^{\prime}, y^{\prime}\right)=\frac{c_{n}}{r^{\frac{1}{2}}\left|r x^{\prime}-y^{\prime}\right|^{n-2}} .
$$

Let us also define $\widetilde{S}_{0}=M_{r^{\frac{n-1}{2}}} S_{0} M_{r^{-\frac{n-1}{2}}}$.

Lemma 5.1. The operator $\widetilde{S}_{0}$ has Mellin convolution kernel

$$
\widetilde{h}\left(r, x^{\prime}, y^{\prime}\right)=\frac{c_{n}}{\left|r^{\frac{1}{2}} x^{\prime}-r^{-\frac{1}{2}} y^{\prime}\right|^{n-2}},
$$

where $x^{\prime}, y^{\prime} \in \partial \omega$. 
Proof. By Proposition 3.6, the operator $\widetilde{S_{0}}$ has Mellin convolution kernel

$$
\tilde{h}\left(r, x^{\prime}, y^{\prime}\right)=r^{\frac{n-1}{2}} h\left(r, x^{\prime}, y^{\prime}\right)=\frac{c_{n} r^{\frac{n-1}{2}}}{r^{\frac{1}{2}}\left|r x^{\prime}-y^{\prime}\right|^{n-2}}=\frac{c_{n}}{\left|r^{\frac{1}{2}} x^{\prime}-r^{-\frac{1}{2}} y^{\prime}\right|^{n-2}} .
$$

Remark 5.2. So $\widetilde{h}$ is obtained as a boundary value of $\widetilde{\phi}_{0}$.

Lemma 5.3. The operator $\widetilde{S}_{0}$ is symmetric.

Proof. Let $\widetilde{S}_{0}^{*}$ be the formal adjoint of $\widetilde{S}_{0}$. Then the convolution kernel of $\widetilde{S}_{0}^{*}$ is

$$
\widetilde{h}^{*}\left(r, x^{\prime}, y^{\prime}\right):=\widetilde{h}\left(r^{-1}, y^{\prime}, x^{\prime}\right) .
$$

From the expression of $\widetilde{h}$ in Lemma 5.1 , we have $\widetilde{h}^{*}=\widetilde{h}$. Hence $\widetilde{S}_{0}$ is symmetric.

\section{MAPping PROPERTIES OF $K$ AND $S$}

Let $m \in \mathbb{Z}_{+}, \alpha \in \mathbb{Z}_{+}^{n}$ be a multi-index. We define the $m$-th Sobolev space on $\Omega$ with weight $r$ and index $a$ by

$$
\mathcal{K}_{a}^{m}(\Omega)=\left\{u \in L_{\mathrm{loc}}^{2}(\Omega, d x), r^{|\alpha|-a} \partial^{\alpha} u \in L^{2}(\Omega, d x), \text { for all }|\alpha| \leqslant m\right\} .
$$

The norm on $\mathcal{K}_{a}^{m}(\Omega)$ is $\|u\|_{\mathcal{K}_{a}^{m}(\Omega)}^{2}:=\sum_{|\alpha| \leqslant m}\left\|r^{|\alpha|-a} \partial^{\alpha} u\right\|_{L^{2}(\Omega, d x)}^{2}$. By Theorem 5.6 in [1], this norm is equivalent to $\|u\|_{m, a}^{2}:=\sum_{|\alpha| \leqslant m}\left\|r^{-a}(r \partial)^{\alpha} u\right\|_{L^{2}(\Omega, d x)}^{2}$, where $(r \partial)^{\alpha}=$ $\left(r \partial_{1}\right)^{\alpha_{1}}\left(r \partial_{2}\right)^{\alpha_{2}} \cdots\left(r \partial_{n}\right)^{\alpha_{n}}$. Clearly, we have that $r^{t} \mathcal{K}_{a}^{m}(\Omega) \cong \mathcal{K}_{a+t}^{m}(\Omega)$. In general, this isomorphism may not be an isometry.

Proposition 6.1. We have, for all $m \in \mathbb{Z}$,

$$
\mathcal{K}_{\frac{n}{2}}^{m}(\Omega) \cong H^{m}(\Omega, g),
$$

where the metric $g$ is $\left(r^{-1} d r\right)^{2}+\left(d x^{\prime}\right)^{2}$.

Proof. The result follows from Proposition 5.7 in [1], and can also be easily checked by direct computations.

The identification given above allows us to define weighted Sobolev spaces on the boundary $\mathcal{K}_{a}^{m}(\partial \Omega)$. For more details, see [1].

Proposition 6.2. For $m \in \mathbb{Z}_{+}$, we have the following identification:

$$
\mathcal{K}_{\frac{n-1}{2}}^{m}(\partial \Omega) \cong H^{m}(\partial \Omega, g)
$$

Proof. The result follows from Definition 5.8 in [1], and can also be easily checked by direct computations.

To study the mapping properties of operators in $\mathcal{R}_{k}$, we need to isolate their singularity near 1 . For this purpose, let us now choose a smooth cutoff function

$$
\chi\left(r, x^{\prime}, y^{\prime}\right)=\chi(r)= \begin{cases}1 & \text { if }|r-1| \leqslant \delta \\ 0 & \text { if }|r-1| \geqslant 2 \delta .\end{cases}
$$

First, let us recall the explicit form of some of the operators that we will need below. By Lemma 4.7 and Proposition 3.6, the operator $M_{r^{a}} \widetilde{K} M_{r^{-a}}$ has convolution kernel

$$
\widetilde{k}_{a}\left(r, x^{\prime}, y^{\prime}\right)=r^{a} \widetilde{k}\left(r, x^{\prime}, y^{\prime}\right)=-\frac{1}{\omega_{n}} \frac{r^{a+\frac{1}{2}} x^{\prime} \cdot \nu\left(y^{\prime}\right)}{\left|r^{\frac{1}{2}} x^{\prime}-r^{-\frac{1}{2}} y^{\prime}\right|^{n}} .
$$


By Lemma 5.1 and Proposition 3.6 $M_{r_{b}} \widetilde{S}_{0} M_{r^{-b}}$ is also a Mellin convolution operator with kernel

$$
\widetilde{h}_{b}\left(r, x^{\prime}, y^{\prime}\right)=r^{b} \widetilde{h}\left(r, x^{\prime}, y^{\prime}\right)=\frac{c_{n} r^{b}}{\left|r^{\frac{1}{2}} x^{\prime}-r^{-\frac{1}{2}} y^{\prime}\right|^{n-2}} .
$$

Proposition 6.3. Let $\widetilde{k}_{a}$ and $\widetilde{h}_{b}$ be given by Equations (21) and (22) above.

(i) If $-\frac{n+1}{2}<a<\frac{n-1}{2}$, then $(1-\chi) \widetilde{k}_{a} \in C^{\infty}([0, \infty] \times \partial \omega \times \partial \omega)$.

(ii) If $-\frac{n-2}{2}<b<\frac{n-2}{2}$, then $(1-\chi) \widetilde{h}_{b} \in C^{\infty}([0, \infty] \times \partial \omega \times \partial \omega)$.

Proof. Let $r(z)=\frac{1+z}{1-z}$. Then $r:[-1,1] \rightarrow[0, \infty]$ is a map with $r(-1)=0$ and $r(1)=\infty$. Under this changes of variables, the differential operator $r \partial_{r}$ (acting on functions on $\left.\mathbb{R}^{+}\right)$corresponds to the differential operator $D_{z}=\frac{1}{2}(1+z)(1-z) \partial_{z}$ (acting on functions on $[-1,1])$. The pullback of the differential structure on $(0, \infty)$ defines a differential structure on $[-1,1]$ with smooth functions $\mathcal{C}_{b}^{\infty}([-1,1])$. More precisely, $\mathcal{C}_{b}^{\infty}([-1,1])$ consists of continuous functions $\xi:[-1,1] \rightarrow \mathbb{C}$ such that $D_{z}^{k} \xi$ is also continuous on $[-1,1]$ for all $k$. Clearly, we have

$$
D_{z}\left(\frac{1+z}{1-z}\right)^{m}=\frac{1}{2}(1+z)(1-z) \partial_{z}\left(\frac{1+z}{1-z}\right)^{m}=m\left(\frac{1+z}{1-z}\right)^{m} .
$$

Moreover, we calculate

$$
\begin{aligned}
g_{a}\left(z, x^{\prime}, y^{\prime}\right) & :=(1-\chi(r(z))) \tilde{k}_{a}\left(r(z), x^{\prime}, y^{\prime}\right) \\
& =-\frac{1}{\omega_{n}}\left(\frac{1+z}{1-z}\right)^{\frac{n+1}{2}+a} \frac{x^{\prime} \cdot \nu\left(y^{\prime}\right)\left(1-\chi\left(\frac{1+z}{1-z}\right)\right)}{\left|\left(\frac{1+z}{1-z}\right) x^{\prime}-y^{\prime}\right|^{n}} .
\end{aligned}
$$

If $-\frac{n+1}{2}<a<\frac{n-1}{2}$, the function $g_{a}$ can be extended to -1 and 1 . Applying the operator $D_{z}$ to the function $g_{a}$, we obtain a function of the same type as $g_{a}$. By iterating differentiation, we find that $g_{a} \in \mathcal{C}_{b}^{\infty}([-1,1]) \times \partial \omega \times \partial \omega$, so $(1-\chi) \widetilde{k}_{a} \in$ $C^{\infty}([0, \infty] \times \partial \omega \times \partial \omega)$. Similarly, we compute

$$
\begin{aligned}
(1-\chi(r(z))) \tilde{h}_{b}\left(r(z), x^{\prime}, y^{\prime}\right)=\frac{c_{n}\left(\frac{1+z}{1-z}\right)^{b}\left(1-\chi\left(\frac{1+z}{1-z}\right)\right)}{\left|\left(\frac{1+z}{1-z}\right)^{\frac{1}{2}} x^{\prime}-\left(\frac{1+z}{1-z}\right)^{-\frac{1}{2}} y^{\prime}\right|^{n-2}} \\
\quad=c_{n}\left(\frac{1+z}{1-z}\right)^{\frac{n-2}{2}+b} \frac{1-\chi\left(\frac{1+z}{1-z}\right)}{\left|\left(\frac{1+z}{1-z}\right) x^{\prime}-y^{\prime}\right|^{n-2}} .
\end{aligned}
$$

For the same reason, $(1-\chi) \tilde{h}_{b} \in C^{\infty}([0, \infty] \times \partial \omega \times \partial \omega)$, provided that $-\frac{n-2}{2}<b<$ $\frac{n-2}{2}$.

Lemma 6.4. Let $\widetilde{k}_{a}$ and $\widetilde{h}_{b}$ be given by Equations (21) and (22) above.

(i) If $-\frac{n+1}{2}<a<\frac{n-1}{2}$, choose $\epsilon_{1}>0$ so that $a+\epsilon_{1}<n-1$ and $a-\epsilon_{1}>-1$, then there exists some constant $C_{1}=C_{1}\left(\epsilon_{1}, i, j, l\right)$, such that $\sup _{\left(r, x^{\prime}, y^{\prime}\right) \in \mathbb{R}^{+} \times \partial \omega \times \partial \omega}\left(r+\frac{1}{r}\right)^{\epsilon_{1}}\left|\left(r \partial_{r}\right)^{i} \Delta_{\partial \omega, x^{\prime}}^{j} \Delta_{\partial \omega, y^{\prime}}^{l}(1-\chi) \tilde{k}_{a}\left(r, x^{\prime}, y^{\prime}\right)\right| \leqslant C_{1} ;$

As a consequence, $(1-\chi) \tilde{k}_{a} \in \mathcal{R}_{-\infty}$. 
(ii) If $-\frac{n-2}{2}<b<\frac{n-2}{2}$, choose $\epsilon_{2}>0$ so that $b+\epsilon_{2}<\frac{n-2}{2}$, then there exists some constant $C_{2}=C_{2}\left(\epsilon_{2}, i, j, l\right)$, such that

$$
\sup _{\left(r, x^{\prime}, y^{\prime}\right) \in \mathbb{R}^{+} \times \partial \omega \times \partial \omega}\left(r+\frac{1}{r}\right)^{\epsilon_{2}}\left|\left(r \partial_{r}\right)^{i} \Delta_{\partial \omega, x^{\prime}}^{j} \Delta_{\partial \omega, y^{\prime}}^{l}(1-\chi) \widetilde{h}_{b}\left(r, x^{\prime}, y^{\prime}\right)\right| \leqslant C_{2} .
$$

As a consequence, $(1-\chi) \widetilde{h}_{b} \in \mathcal{R}_{-\infty}$.

Proof. This is a consequence of Proposition 6.3.

We are ready to prove one of our main technical results.

Theorem 6.5. Let $\widetilde{K}=M_{r^{\frac{n-1}{2}}} K M_{r^{-\frac{n-1}{2}}}$ and $\widetilde{S_{0}}=M_{r^{\frac{n-1}{2}}} S_{0} M_{r^{-\frac{n-1}{2}}}$, then

(i) If $-\frac{n+1}{2}<a<\frac{n-1}{2}$, then $M_{r^{a}} \tilde{K} M_{r^{-a}} \in \mathcal{R}_{-1}$;

(ii) If $-\frac{n-2}{2}<b<\frac{n-2}{2}$, then $M_{r^{b}} \widetilde{S}_{0} M_{r^{-b}} \in \mathcal{R}_{-1}$.

Proof. Using the cutoff function $\chi$ in Equation (20), we write

$$
\widetilde{k}_{a}=(1-\chi) \widetilde{k}_{a}+\chi \widetilde{k}_{a} .
$$

By Lemma 6.4 , we have $(1-\chi) \widetilde{k}_{a} \in \mathcal{R}_{-\infty}$. Since $\chi \widetilde{k}$ is properly supported, dilation invariant, and of order -1 , by Corollary 3.7 , we have $\chi \widetilde{k}_{a} \in \Psi_{\text {prop }}^{-1}\left(\mathbb{R}^{+} \times \partial \omega\right)^{\mathbb{R}^{+}}$. Therefore, we have $M_{r^{a}} K M_{r^{-a}} \in \mathcal{R}_{-\infty}+\Psi_{\text {prop }}^{-1}\left(\mathbb{R}^{+} \times \partial \omega\right)^{\mathbb{R}^{+}}=\mathcal{R}_{-1}$. The same argument can be applied to $M_{r^{b}} \widetilde{S}_{0} M_{r^{-b}}$.

Similarly, we have the following.

Theorem 6.6. The following operators are well-defined and continuous for all $m \in \mathbb{Z}:$

(i) $M_{r^{a}} \widetilde{K} M_{r^{-a}}: \mathcal{K}_{\frac{n-1}{2}}^{m}(\partial \Omega) \rightarrow \mathcal{K}_{\frac{n-1}{2}}^{m+1}(\partial \Omega)$, for $-\frac{n+1}{2}<a<\frac{n-1}{2}$;

(ii) $M_{r^{b}} \widetilde{S}_{0} M_{r^{-b}}: \mathcal{K}_{\frac{n-1}{2}}^{m}(\partial \Omega) \rightarrow \mathcal{K}_{\frac{n-1}{2}}^{m+1}(\partial \Omega)$ for $-\frac{n-2}{2}<b<\frac{n-2}{2}$.

Proof. The results follow immediately from Theorem 6.5, Theorem 6.2, and Corollary 2.12 .

For the operators of interest, we obtain the following.

Corollary 6.7. We have the following mapping properties:

(i) if $-1<a<n-1$ and $m \in \mathbb{Z}$, then

$$
K: \mathcal{K}_{\frac{n-1}{2}-a}^{m}(\partial \Omega) \rightarrow \mathcal{K}_{\frac{n-1}{2}-a}^{m+1}(\partial \Omega)
$$

(ii) if $\frac{1}{2}<b<n-\frac{3}{2}$ and $m \in \mathbb{Z}$, then

$$
S_{0}: \mathcal{K}_{\frac{n-1}{2}-b}^{m}(\partial \Omega) \rightarrow \mathcal{K}_{\frac{n-1}{2}-b}^{m+1}(\partial \Omega)
$$

(iii) if $\frac{1}{2}<b<n-\frac{3}{2}$ and $m \in \mathbb{Z}$, then

$$
S: \mathcal{K}_{\frac{n-2}{2}-b}^{m}(\partial \Omega) \rightarrow \mathcal{K}_{\frac{n}{2}-b}^{m+1}(\partial \Omega) .
$$

Proof. The conclusions follow from the relations $\widetilde{K}=M_{r^{\frac{n-1}{2}}} K M_{r^{-\frac{n-1}{2}}}, \widetilde{S}_{0}=$ $M_{r^{\frac{n-1}{2}}} S_{0} M_{r^{-\frac{n-1}{2}}}$, and $S_{0}=M_{r^{-\frac{1}{2}}} S M_{r^{-\frac{1}{2}}}$.

We shall also need the following in order to prove that the pointwise invertibility of the Mellin transform implies the global invertibility. 
Lemma 6.8. We have $\widetilde{K}, \widetilde{S}_{0} \in \mathcal{A}$, where $\mathcal{A}$ is defined in Section 3. As a result, $K, S_{0} \in \mathcal{A}$.

Proof. This is a consequence of Theorem 6.5 and Definition 3.11.

\section{INVERTIBILITY OF LAYER POTENTIALS}

In this section, we will use functional analytic argument to reduce global invertibility of $\frac{1}{2}+K$ and $S$, acting on suitable Sobolev spaces, to the invertibility of their Mellin transform for each $t$. To this end, we will use that $\widetilde{S}_{0}$ and $\widetilde{K}$ are in $\mathcal{R}_{-1}$.

We now come to one of our main results, which identifies the Mellin transform of the double layer potential operator $K(\Delta, E ; \Omega)$ of $\Delta$ on the cone $\Omega:=\mathbb{R}^{+} \omega$ in terms of the double layer potential of $\Delta_{\omega}-t^{2}-\frac{(n-2)^{2}}{4}$ on $\omega$. Recall that $E$ is the standard fundamental solution of $\Delta$, that $\phi_{0}$ is the Mellin convolution kernel of $\Phi_{0}$, and that $\widetilde{\phi}_{0}$ is the Mellin convolution kernel of $\widetilde{\Phi}_{0}:=M_{r^{\frac{n}{2}}} \Phi_{0} M_{r^{-\frac{n}{2}}}$.

Theorem 7.1. Let $-\frac{n-2}{2}<\Im(t)<\frac{n-2}{2}$. We have

$$
(\mathcal{M} K)(t-i / 2)=\mathcal{M}^{\sharp} \tilde{K}(t-i / 2)=K\left(\Delta_{\omega}-t^{2}-\frac{(n-2)^{2}}{4}, \mathcal{M}^{\sharp} \widetilde{\phi}_{0}(t) ; \omega\right) .
$$

Let us notice also that $\mathcal{M}^{\sharp} \widetilde{\phi}_{0}=\mathcal{M} \phi_{0}$, and hence

$$
(\mathcal{M K})(t-i / 2)=K\left(\Delta_{\omega}-t^{2}-\frac{(n-2)^{2}}{4}, \mathcal{M} \phi_{0}(t) ; \omega\right) .
$$

In the following, we shall denote

$$
c(t):=-t^{2}-\frac{(n-2)^{2}}{4} .
$$

Proof. It is clear from the definition and Lemma 3.10 that $\mathcal{M} K=\mathcal{M}^{\sharp} \tilde{K}$. Let and $L_{t}=\Delta_{\omega}+c(t), c(t):=-t^{2}-\frac{(n-2)^{2}}{4}$, as above. By Corollary 4.6, for each fixed $t$ satisfying $-\frac{n-2}{2}<\Im(t)<\frac{n-2}{2}$, the function $\mathcal{M}^{\sharp} \widetilde{\phi}_{0}\left(t, x^{\prime}, y^{\prime}\right)$ is a fundamental solution for the elliptic operator $L_{t}=\Delta_{\omega}+c(t)$ on $\omega \subset S^{n-1}$. Furthermore, by Corollaries 4.3(iii) and 4.6, the double layer potential associated to $L_{t}, \mathcal{M}^{\sharp} \widetilde{\phi}_{0}\left(t, x^{\prime}, y^{\prime}\right)$, and $\omega$ is

$$
\begin{aligned}
& K\left(L_{t}, \mathcal{M}^{\sharp} \widetilde{\phi}_{0}(t) ; \omega\right)=\partial_{\nu_{y^{\prime}}} \mathcal{M}^{\sharp} \widetilde{\phi}_{0}\left(t, x^{\prime}, y^{\prime}\right)=\int_{0}^{\infty} r^{-i t} \partial_{\nu_{y^{\prime}}} \widetilde{\phi}_{0}\left(r, x^{\prime}, y^{\prime}\right) \frac{d r}{r} \\
&=\int_{0}^{\infty} r^{-i t} \partial_{\nu_{y^{\prime}}} \frac{c_{n}}{\left|r^{\frac{1}{2}} x^{\prime}-r^{-\frac{1}{2}} y^{\prime}\right|^{n-2}} \frac{d r}{r}=-\frac{1}{\omega_{n}} \int_{0}^{\infty} r^{-i t} \frac{x^{\prime} \cdot \nu\left(y^{\prime}\right)}{\left|r^{\frac{1}{2}} x^{\prime}-r^{-\frac{1}{2}} y^{\prime}\right|^{n}} \frac{d r}{r},
\end{aligned}
$$

since $-1 / \omega_{n}=(n-2) c_{n}$.

On the other hand, Lemma 4.7 gives

$$
\begin{aligned}
\mathcal{M}^{\sharp} \widetilde{K}\left(t-i / 2, x^{\prime}, y^{\prime}\right) & =\int_{0}^{\infty} r^{-i t-1 / 2} \widetilde{k}\left(r, x^{\prime}, y^{\prime}\right) \frac{d r}{r} \\
= & -\frac{1}{\omega_{n}} \int_{0}^{\infty} r^{-i t-1 / 2} \frac{r^{\frac{1}{2}} x^{\prime} \cdot \nu\left(y^{\prime}\right)}{\left|r^{\frac{1}{2}} x^{\prime}-r^{-\frac{1}{2}} y^{\prime}\right|^{n}} \frac{d r}{r}=K\left(L_{t}, \mathcal{M}^{\sharp} \widetilde{\phi}_{0}(t) ; \omega\right) .
\end{aligned}
$$

This gives the desired result. 
Theorem 7.2. Let $-\frac{n-2}{2}<\Im(t)<\frac{n-2}{2}$. We have

$$
\mathcal{M} S_{0}(t)=\mathcal{M}^{\sharp} \widetilde{S}_{0}(t)=S\left(\Delta_{\omega}-t^{2}-\frac{(n-2)^{2}}{4}, \mathcal{M}^{\sharp} \widetilde{\phi}_{0}(t) ; \omega\right) .
$$

Let us notice also that $\mathcal{M}^{\sharp} \widetilde{\phi}_{0}=\mathcal{M} \phi_{0}$, and hence

$$
\begin{aligned}
\mathcal{M} S(t)=S\left(\Delta_{\omega}-t^{2}-\frac{(n-2)^{2}}{4}, \mathcal{M}^{\sharp} \widetilde{\phi}_{0}(t) ; \omega\right) & \\
& =S\left(\Delta_{\omega}-t^{2}-\frac{(n-2)^{2}}{4}, \mathcal{M} \phi_{0}(t) ; \omega\right) .
\end{aligned}
$$

Proof. The relation $\mathcal{M} S_{0}=\mathcal{M}^{\sharp} \widetilde{S}_{0}$ follows from definitions. Let $L_{t}=\Delta_{\omega}+c(t)$, with $c(t)=-t^{2}-\frac{(n-2)^{2}}{4}$ as before. By Proposition 4.5, for each fixed $t$ satisfying $-\frac{n-2}{2}<\Im(t)<\frac{n-2}{2}$, the function $\mathcal{M}^{\sharp} \tilde{\phi}_{0}\left(t, x^{\prime}, y^{\prime}\right), x^{\prime}, y^{\prime} \in \omega$, is a fundamental solution for the elliptic operator $L_{t}=\Delta_{\omega}+c(t)$ on $\omega \subset S^{n-1}$. Hence, the single layer potential associated to $L_{t}, \mathcal{M} \phi_{0}\left(t, x^{\prime}, y^{\prime}\right)$, and $\omega$ is simply the operator with kernel $\mathcal{M}^{\sharp} \widetilde{\phi}_{0}\left(t, x^{\prime}, y^{\prime}\right), x^{\prime}, y^{\prime} \in \partial \omega$, and hence

$$
S\left(L_{t}, \mathcal{M}^{\sharp} \tilde{\phi}_{0}(t) ; \omega\right)=\mathcal{M}^{\sharp} \widetilde{\phi}_{0}\left(t, x^{\prime}, y^{\prime}\right)=\int_{0}^{\infty} r^{-i t} \frac{c_{n}}{\left|r^{\frac{1}{2}} x^{\prime}-r^{\frac{1}{2}} y^{\prime}\right|^{n-2}} \frac{d r}{r} .
$$

Similarly, recall that $\tilde{h}\left(t, x^{\prime}, y^{\prime}\right)$ is the convolution kernel of $\widetilde{S}_{0}$, and hence

$$
\begin{aligned}
\mathcal{M}^{\sharp} \widetilde{S}_{0}\left(t, x^{\prime}, y^{\prime}\right) & =\int_{0}^{\infty} r^{-i t} \widetilde{\phi}_{0}\left(t, x^{\prime}, y\right) \frac{d r}{r} \\
& =\int_{0}^{\infty} r^{-i t} \frac{c_{n}}{\left|r^{\frac{1}{2}} x^{\prime}-r^{\frac{1}{2}} y^{\prime}\right|^{n-2}} \frac{d r}{r},
\end{aligned}
$$

so the proof is complete.

In the next two theorems, in order to apply theorems in [35] to obtain invertibility of $\frac{1}{2}+K$ and $S$, we need to assume that $\omega \subset S^{n-1}$ is connected.

Theorem 7.3. Assume $\omega$ is connected. Let $-\frac{n-2}{2}<\Im(t)<\frac{n-2}{2}$. Then the operator $\frac{1}{2}+\mathcal{M} K(t)=\frac{1}{2}+\mathcal{M}^{\sharp} \widetilde{K}(t): H^{m}(\partial \omega) \rightarrow H^{m}(\partial \omega)$ is invertible. In particular, if $-\frac{n-2}{2}<a<\frac{n-2}{2}$, then

$$
\frac{1}{2}+\widetilde{K}: \mathcal{K}_{\frac{n-1}{2}-a}^{m}(\partial \Omega) \rightarrow \mathcal{K}_{\frac{n-1}{2}-a}^{m}(\partial \Omega)
$$

is invertible for all $m \in \mathbb{Z}$.

Proof. By Theorem 7.1, we can apply the double layer potential theory to the operator $\Delta_{\omega}+c(t), c(t)=-t^{2}-\frac{(n-2)^{2}}{4}$, with domain $\omega$. First, we notice that $\Delta_{\omega}+c(t): H_{0}^{1}(\omega) \rightarrow H^{-1}(\omega)$ is invertible for all $t$ satisfying $-\frac{n-2}{2}<\Im(t)<\frac{n-2}{2}$. Since $\omega$ has smooth boundary, by Proposition 6.3 in Chapter 3 in [35], we obtain that $\frac{1}{2}+\mathcal{M} K(t): H^{m}(\partial \omega) \rightarrow H^{m}(\partial \omega)$ is invertible for each $t$ satisfying $-\frac{n-2}{2}<$ $\Im(t)<\frac{n-2}{2}$. By Lemma 6.8 and Theorem 3.12, the operator $\frac{1}{2}+\widetilde{K}: \mathcal{K}_{\frac{n-1}{2}-a}^{m}(\partial \Omega) \rightarrow$ $\mathcal{K}_{\frac{n-1}{2}-a}^{m}(\partial \Omega)$ is invertible.

We now prove analogous results for the single layer potential operator. 
Theorem 7.4. Assume $\omega$ is connected. Let $-\frac{n-2}{2}<\Im(t)<\frac{n-2}{2}$. Then the operator $\left(\mathcal{M} S_{0}\right)(t)=\mathcal{M}^{\sharp} \widetilde{S}_{0}(t): H^{m}(\partial \omega) \rightarrow H^{m+1}(\partial \omega)$ is invertible. In particular, if $-\frac{n-2}{2}<b<\frac{n-2}{2}$ then

$$
\widetilde{S}_{0}: \mathcal{K}_{\frac{n-1}{2}-b}^{m}(\partial \Omega) \rightarrow \mathcal{K}_{\frac{n-1}{2}-b}^{m+1}(\partial \Omega)
$$

is invertible for each $m \in \mathbb{Z}$.

Proof. By Theorem 7.2, we can apply the single layer potential theory to $\Delta_{\omega}+c(t)$ with domain $\omega$. Since $\omega$ has smooth boundary, by Proposition 6.5 in Chapter 3 in [35], we know that $\left(\mathcal{M} S_{0}\right)(t)=\mathcal{M}^{\sharp} \widetilde{S}_{0}(t): H^{m}(\partial \omega) \rightarrow H^{m+1}(\partial \omega)$ is invertible for each $t$ satisfying $-\frac{n-2}{2}<\Im(t)<\frac{n-2}{2}$. By Lemma 6.8 and Theorem 3.12 again, the operator $\widetilde{S}_{0}: \mathcal{K}_{\frac{n-1}{2}-b}^{m}(\partial \Omega) \rightarrow \mathcal{K}_{\frac{n-1}{2}-b}^{m+1}(\partial \Omega)$ is invertible.

Corollary 7.5. Let $m \in \mathbb{Z}$ be arbitrary.

(1) $\frac{1}{2}+K: \mathcal{K}_{\frac{n-1}{2}-a}^{m}(\partial \Omega) \rightarrow \mathcal{K}_{\frac{n-1}{2}-a}^{m}(\partial \Omega)$ is invertible for $\frac{1}{2}<a<n-\frac{3}{2}$.

(2) $S_{0}: \mathcal{K}_{\frac{n-1}{2}-b}^{m}(\partial \Omega) \rightarrow \mathcal{K}_{\frac{n-1}{2}-b}^{m+1}(\partial \Omega)$ is invertible for $\frac{1}{2}<b<n-\frac{3}{2}$.

(3) $S: \mathcal{K}_{\frac{n-2}{2}-b}^{m}(\partial \Omega) \rightarrow \mathcal{K}_{\frac{n}{2}-b}^{m+1}(\partial \Omega)$ is invertible for $\frac{1}{2}<b<n-\frac{3}{2}$.

Proof. Since $\widetilde{K}=M_{r^{\frac{n-1}{2}}} K M_{r^{-\frac{n-1}{2}}}$ and $\widetilde{S}_{0}=M_{r^{\frac{n-1}{2}}} S_{0} M_{r^{-\frac{n-1}{2}}}$, the results are direct consequences of the preceding two theorems.

\section{REFERENCES}

[1] C. Bacuta, A. Mazzucato, V. Nistor, and L. Zikatanov. Interface and mixed boundary value problems on $n$-dimensional polyhedral domain. Doc. Math., 15:687-745, 2010.

[2] J. Bremer and V. Rokhlin. Efficient discretization of Laplace boundary integral equations on polygonal domains. J. Comput. Phys., 229(7):2507-2525, 2010.

[3] M. Costabel. Boundary integral operators on curved polygons. Ann. Mat. Pura Appl. (4), 133:305-326, 1983.

[4] R. Courant and D. Hilbert. Methods of mathematical physics. Vol. II. Wiley Classics Library. John Wiley \& Sons Inc., New York, 1989. Partial differential equations, Reprint of the 1962 original, A Wiley-Interscience Publication.

[5] Y. Egorov and B.-W. Schulze. Pseudo-differential operators, singularities, applications, volume 93 of Operator Theory: Advances and Applications. Birkhäuser Verlag, Basel, 1997.

[6] J. Elschner. The double layer potential operator over polyhedral domains. I. Solvability in weighted Sobolev spaces. Appl. Anal., 45(1-4):117-134, 1992.

[7] L. Evans. Partial differential equations, volume 19 of Graduate Studies in Mathematics. American Mathematical Society, Providence, RI, 1998.

[8] E. Fabes, M. Jodeit, and J. Lewis. Double layer potentials for domains with corners and edges. Indiana Univ. Math. J., 26(1):95-114, 1977.

[9] E. Fabes, M. Jodeit, and N. Rivière. Potential techniques for boundary value problems on $C^{1}$-domains. Acta Math., 141(3-4):165-186, 1978.

[10] G. Folland. Introduction to partial differential equations. Princeton University Press, Princeton, NJ, second edition, 1995.

[11] L. Hörmander. The analysis of linear partial differential operators. III, volume 274 of Grundlehren der Mathematischen Wissenschaften [Fundamental Principles of Mathematical Sciences]. Springer-Verlag, Berlin, 1985. Pseudodifferential operators.

[12] D. Jerison and C. Kenig. The Dirichlet problem in nonsmooth domains. Ann. of Math. (2), 113(2):367-382, 1981.

[13] D. Jerison and C. Kenig. The Neumann problem on Lipschitz domains. Bull. Amer. Math. Soc. (N.S.), 4(2):203-207, 1981.

[14] D. Jerison and C. Kenig. The inhomogeneous Dirichlet problem in Lipschitz domains. J. Funct. Anal., 130(1):161-219, 1995. 
[15] D. Kapanadze and B.-W. Schulze. Boundary-contact problems for domains with conical singularities. J. Differential Equations, 217(2):456-500, 2005.

[16] R. Kellogg. Singularities in interface problems. In Numerical Solution of Partial Differential Equations, II (SYNSPADE 1970) (Proc. Sympos., Univ. of Maryland, College Park, Md., 1970), pages 351-400. Academic Press, New York, 1971.

[17] C. Kenig. Recent progress on boundary value problems on Lipschitz domains. In Pseudodifferential operators and applications (Notre Dame, Ind., 1984), volume 43 of Proc. Sympos. Pure Math., pages 175-205. Amer. Math. Soc., Providence, RI, 1985.

[18] C. Kenig and J. Pipher. The Neumann problem for elliptic equations with nonsmooth coefficients. II. Duke Math. J., 81(1):227-250 (1996), 1995. A celebration of John F. Nash, Jr.

[19] V. Kondrat'ev. Boundary value problems for elliptic equations in domains with conical or angular points. Trudy Moskov. Mat. Obšč., 16:209-292, 1967.

[20] R. Kress. Linear integral equations, volume 82 of Applied Mathematical Sciences. SpringerVerlag, New York, second edition, 1999.

[21] R. Lauter, B. Monthubert, and V. Nistor. Spectral invariance for certain algebras of pseudodifferential operators. J. Inst. Math. Jussieu, 4(3):405-442, 2005.

[22] J. Lewis. Layer potentials for elastostatics and hydrostatics in curvilinear polygonal domains. Trans. Amer. Math. Soc., 320(1):53-76, 1990.

[23] J. Lewis and C. Parenti. Pseudodifferential operators of Mellin type. Comm. Partial Differential Equations, 8(5):477-544, 1983.

[24] V. Maz'ya. Boundary integral equations. In Analysis, IV, volume 27 of Encyclopaedia Math. Sci., pages 127-222. Springer, Berlin, 1991.

[25] V. Maz'ya and J. Rossmann. Elliptic equations in polyhedral domains, volume 162 of Mathematical Surveys and Monographs. American Mathematical Society, Providence, RI, 2010.

[26] W. McLean. Strongly elliptic systems and boundary integral equations. Cambridge University Press, Cambridge, 2000.

[27] R. Melrose. The Atiyah-Patodi-Singer index theorem, volume 4 of Research Notes in Mathematics. A K Peters Ltd., Wellesley, MA, 1993.

[28] D. Mitrea and I. Mitrea. On the Besov regularity of conformal maps and layer potentials on nonsmooth domains. J. Funct. Anal., 201(2):380-429, 2003.

[29] D. Mitrea, M. Mitrea, and J. Pipher. Vector potential theory on nonsmooth domains in $\mathbf{R}^{3}$ and applications to electromagnetic scattering. J. Fourier Anal. Appl., 3(2):131-192, 1997.

[30] I. Mitrea. On the spectra of elastostatic and hydrostatic layer potentials on curvilinear polygons. J. Fourier Anal. Appl., 8(5):443-487, 2002.

[31] I. Mitrea and M. Mitrea. The Poisson problem with mixed boundary conditions in Sobolev and Besov spaces in non-smooth domains. Trans. Amer. Math. Soc., 359(9):4143-4182 (electronic), 2007.

[32] M. Mitrea and V. Nistor. Boundary value problems and layer potentials on manifolds with cylindrical ends. Czechoslovak Math. J., 57(132)(4):1151-1197, 2007.

[33] M. Mitrea and M. Taylor. Boundary layer methods for Lipschitz domains in Riemannian manifolds. J. Funct. Anal., 163(2):181-251, 1999.

[34] M. Mitrea and M. Taylor. Potential theory on Lipschitz domains in Riemannian manifolds: $L^{P}$ Hardy, and Hölder space results. Comm. Anal. Geom., 9(2):369-421, 2001.

[35] M. Taylor. Pseudodifferential operators. Four Lectures at MSRI, 2008.

[36] M. Taylor. Partial differential equations. II, volume 116 of Applied Mathematical Sciences. Springer-Verlag, New York, 1996. Qualitative studies of linear equations.

[37] G. Verchota. Layer potentials and regularity for the Dirichlet problem for Laplace's equation in Lipschitz domains. J. Funct. Anal., 59(3):572-611, 1984.

[38] G. Verchota and A. Vogel. The multidirectional Neumann problem in $\mathbb{R}^{4}$. Math. Ann., 335(3):571-644, 2006

Pennsylvania State University, Math. Dept., University Park, PA 16802

E-mail address: nistor@math.psu.edu

Pennsylvania State University, Math. Dept., University Park, PA 16802

E-mail address: qiao@math.psu.edu 